\title{
An OWL-Based Mobile GeoBI Context Ontology Enabling Location-Based and Context-Based Reasoning and Supporting Contextual Business Analysis
}

\author{
Belko Abdoul Aziz Diallo, Thierry Badard, Frédéric Hubert, Sylvie Daniel \\ Centre de Recherche en Géomatique, Université Laval, Québec City, Canada \\ Email: belko-abdoul-aziz.diallo.1@ulaval.ca, Thierry.Badard@scg.ulaval.ca, Frederic.Hubert@scg.ulaval.ca, \\ Sylvie.Daniel@scg.ulaval.ca
}

Received 4 January 2015; accepted 26 January 2015; published 30 January 2015

Copyright (C) 2015 by authors and Scientific Research Publishing Inc.

This work is licensed under the Creative Commons Attribution International License (CC BY).

http://creativecommons.org/licenses/by/4.0/

(c) () Open Access

\begin{abstract}
Mobile professionals need to be assisted with suitable mobile GeoBI (Geospatial Business Intelligence) systems, which are able to capture, organize and structure the user's reality into a relevant context model and reason on it. GeoBI context modelling and reasoning are still research issues since there is not yet either a model or a relevant taxonomy regarding GeoBI contextual information. To fill this gap, this paper proposes an extended and detailed OWL-based mobile GeoBI context ontology to provide context-aware applications and users with relevant contextual information and context-based reasoning capabilities. Context quality issues are handled an implementation architecture which is provided.
\end{abstract}

\section{Keywords}

Mobile Geospatial Business Intelligence (GeoBI), Context Modelling, Context Ontology, Context Awareness, Context Reasoning, Location-Based Reasoning

\section{Introduction}

In the today's mobile, global, highly competitive, and technology-based business, mobile professionals deserve to get supported with suitable mobile Decision Support Systems (DSS) that we believe, should be 1) GeoBI (Geospatial Business Intelligence)-enabled to take into account geospatial features, and 2) context-based to cap- 
ture the mobile user's reality. According to [1], about $80 \%$ of businesses' data has a geo-spatial dimension. Thereby, GeoBI realizes an intelligent coupling of GIS tools with Business Intelligence (BI) technologies to suitably exploit, analyse and visualize geo-spatial part of business data (e.g. borders, places, addresses, GPS coordinates, routes, etc.). Moreover, since professional mobility is usually justified by the mobile worker's need to experiment a physical proximity [2] to resources, in order, among other things, to acquire accurate information from what he sees, hears, or senses within his work context, a mobile GeoBI system should help capture, organize and structure contextual information into an appropriate context model that would be exploited with BI data to deliver context-based analytics.

Modelling mobile contexts is still a research issue [3] [4], especially mobile GeoBI contexts, since there is not yet either a model or a relevant taxonomy regarding GeoBI contextual information [5]. To fill this gap, the latter authors proposed "a multilevel mobile GeoBI context". Figure 1 presents that model built upon three hierarchical levels: 1) the context-sharing level enables interaction and information sharing between local and remote contexts; 2) the mobile GeoBI context levels organize context into three hierarchical levels, which fits with human perception of space: personal, ambient and surrounding contexts. Ambient context is a subset of surrounding context and both are composite of GeoBI context and subclasses of "context around the user" as shown in Figure 1 ; 3) the context dimensions structures contextual information into relevant dimensions for BI and mobility: personal, business, spatial, social, technological and environmental contexts. The geospatial $(H, \varepsilon)$ and temporal ( $\xi$ ) pictograms highlight that a mobile GeoBI context is location-based and time-dependent. The model was designed into UML (Unified Modeling Language) to conveniently structure contextual information and was limited to top-level concepts to handle basic challenges regarding context modeling.

In this paper, we propose to extend and detail that top-level UML-based model into an OWL (Web Ontology Language)-based context ontology to provide context-aware applications and users with more specific and precise contextual information and context-based reasoning capabilities.

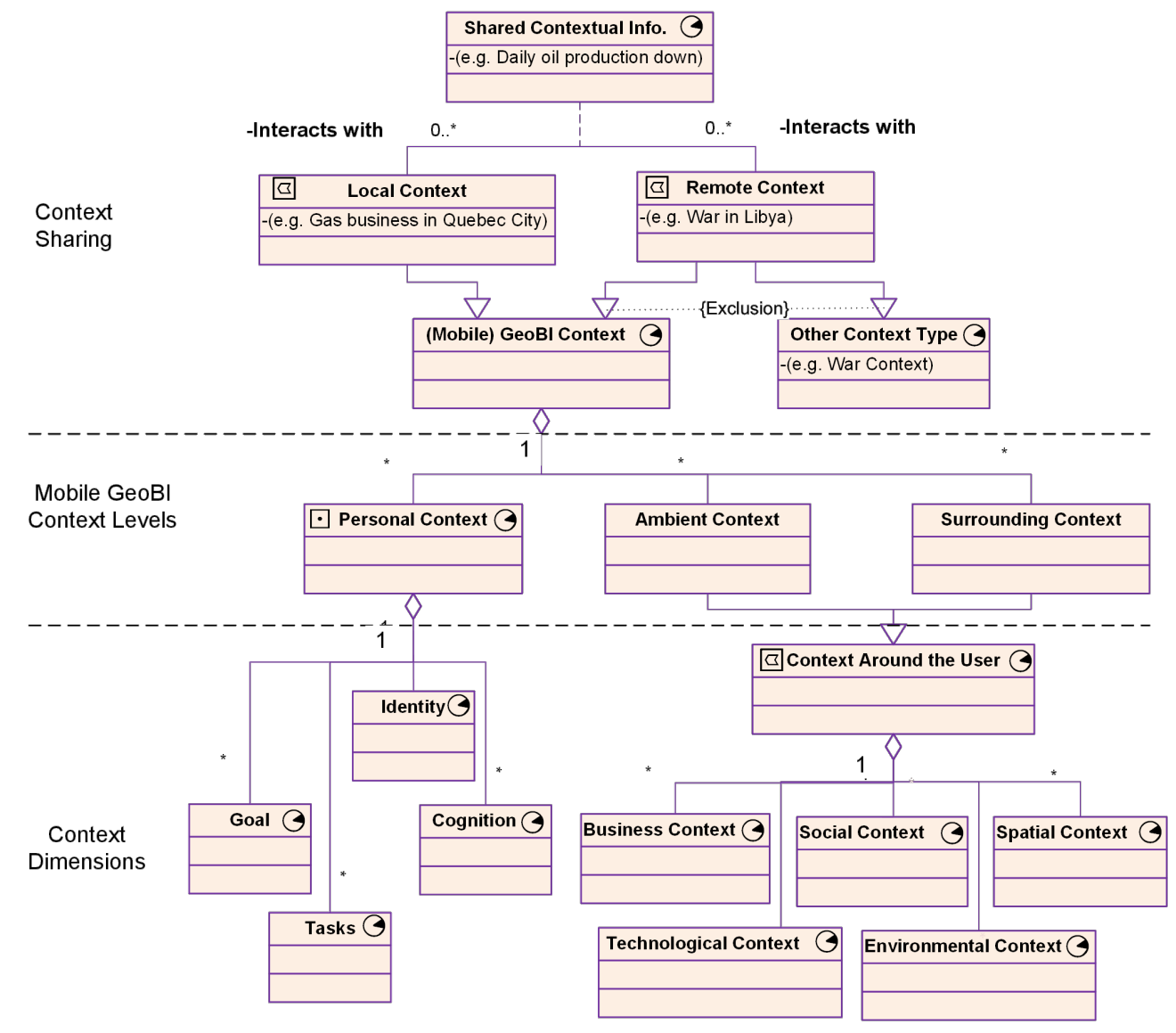

Figure 1. Hierarchical multilevel Mobile GeoBI context model for context sharing and structuring. 
Context reasoning allows applications to infer and discover new contextual information from initial facts. For instance, given that location A (e.g. XYT museum) is located in B (e.g. District 0911) which is located in C (e.g. WYNX sales Zone), if a mobile device is located at XYT museum, then it can derive that the salesman (e.g. Steve) using the device is located in WYNX sales zone. To enable such reasoning, an inferable GeoBI context model is required. In the literature, OWL is usually used to build inferable ontologies [6] and is recognized as more suitable to support knowledge sharing and context reasoning than ER (Entity/relation) and UML [7]. Ontology defines, describes and represents an area of knowledge for the purpose of knowledge-sharing, inferential logic and reasoning [6]. OWL is a text-based language that extends RDF (Resource Description Framework) and RDF-Schema with richer semantics and provides extensibility and compatibility with web standards [8]. To ease its understanding and improve its readability, an OWL-compatible graphical formalism is provided by Cmap Tools COE [9]. This one will be extended with geospatial and temporal pictograms to conveniently describe spatio-temporal concepts and will be then used to design the proposed OWL-based mobile GeoBI context ontology.

The paper is organised as follows: Section 2 highlights the need for GeoBI context-based reasoning and OWL modeling, and presents CmapTools. Section 3 translates the UML-based context model into an OWL-based top-level GeoBI context ontology. Section 4 extends that ontology by detailing each of its context dimensions. Context quality issues are handled in Section 5 and implementation architecture is provided in Section 6.

\section{Need for Context-Based Reasoning and OWL Modelling}

\subsection{Need for Context Reasoning to Support Mobile GeoBI Activities}

To exemplify context reasoning, authors e.g. [6] often consider the transitive relation "is Located In" like in the above example. Let enrich that example by additionally assuming that Steve is a salesman in chief supervising other salesmen which may in turn supervise others. To analyze sales performance achieved by his salesmen in that location, Steve might want to get supported with mobile GeoBI services which are able to handle requests like:

According to the current sales zone I'm in (e.g. WYNX sales Zone), what are the sales performed by the supervisors of the best performing salesman (e.g. John) of this quarter?

Responding to such a request requires contextual information regarding business context (e.g. hierarchy between salesmen), time context (e.g. the quarter), and spatial context (e.g. Steve's current position), and business data regarding salesmen performance (e.g. sales data cube). To determine in which sales region Steve is located based on his current position, i.e. XYT museum, the GeoBI system will have to infer on the transitivity property of the relation "is Located In". The same inference operation is also needed to find out the supervisors of John. Therefore, a GeoBI context ontology allowing such reasoning is expected.

\subsection{Need for OWL Modeling and Context Ontologies}

The contextual information involved in the above example can be modeled by using either UML or OWL. However, whereas OWL is able to express transitive relations ("owl: Transitive Property"), there is no formal way to express that in UML even by resorting to the Object Constraint Language (OCL) provided with UML to define richer constraints. That deficiency of UML/OCL in defining transitive closures is highlighted by [10][12].

In summary, "UML class-based modeling and OWL comprise some constituents that are similar in many respects like classes, associations, properties..." [10], but UML does not "address issues including knowledge sharing and context reasoning" [7] while OWL "approach focuses on context ontology and explores the potential capability of context reasoning" [7].

Figure 2 gives an example of context ontology with reasoning capabilities. That simplified business ontology shows that mobile salesmen Steve and Bill are employed by two different companies operating on the same market. If only the explicit contextual information about Steve and Bill is considered, they will be considered as competitors and will be fighting each other to conquer the Montreal Electronics Market (Figure 2(A)). But, by indicating that the "Owning" relationship between the companies is transitive (symbols =>=>), a simple inference (embracing the domain knowledge) will then reveal that they are in fact working for the same group and should unite to conquer the market against real competitors (Figure 2(B)). Such result is obtained only from de- 


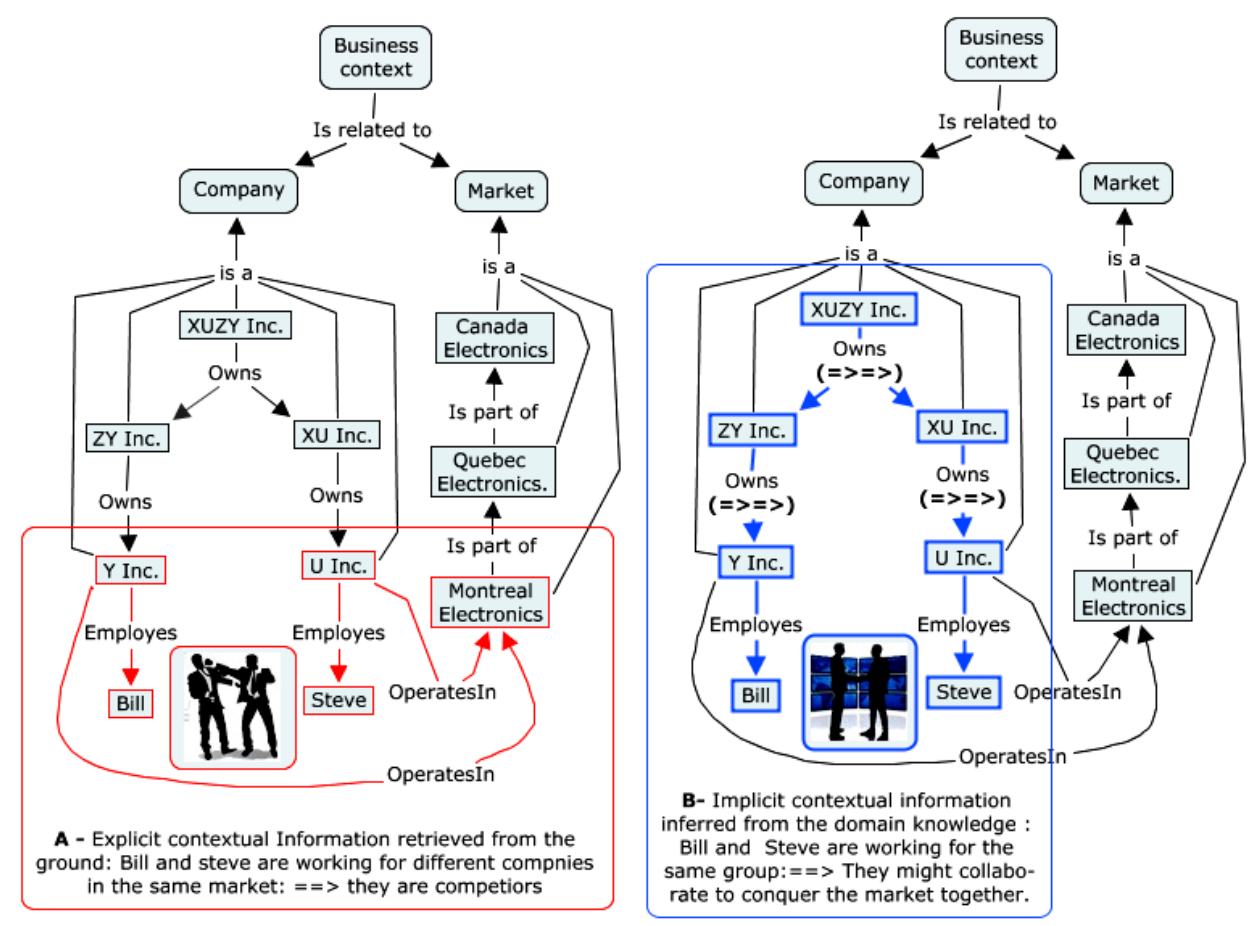

Figure 2. Illustration of context-reasoning based on a simplified business context.

scription logic. The data content are even not yet engaged. In a mobile GeoBI context, being able to get informed of explicit contextual information as well as implicit outcomes it might hide is crucial for decision makers to decide faster but suitably.

\subsection{Cmap Tools COE Graphical Ontology Syntax}

The business context ontology presented in Figure 2 is designed using the OWL-compatible graphical formalism provided by CmapTools COE ontology tool [9]. It is a viewing/editing tool that supports importing and exporting OWL files, and allows graphical drawing and edition of OWL ontologies by using a personalized syntax. That syntax adopts its own lexical and graphical conventions to express OWL and RDF/RDF-S content, rather than mirroring OWL/RDF syntax. Classes (class), individuals (Indiv), and literals (e.g. xsd: string) are nodes with rectangular shapes. Properties are links between: classes, individuals, classes and individuals, individuals and literals, etc. Table 1 underlines some equivalence between CmapTools COE and OWL/RDF syntax. CmapTools COE manual (at ihmc.us) provides further details.

To describe location-based and time-dependent objects, geospatial $(H, \omega, \varepsilon)$ and temporal $(\xi)$ pictograms introduced by [13] and adopted by many others like [14] [15], etc., have been manually integrated to CmapTools graphics. The OWL syntax has been accordingly enriched by using Geo-OWL syntax to handle geospatial issues, and by using "datetime" properties to describe time-dependent classes. "Geo-OWL provides an ontology which closely matches the GeoRSS feature model and which utilizes the existing GeoRSS vocabulary for geographic properties and classes." [16]. The temporal pictogram means that the object has a starting and an ending time properties, whereas geo-spatial pictogram indicates the geometry of a geospatial object. Figure 3 illustrates how they are used in Cmap COE and OWL syntaxes. The involved properties (e.g. begin, end, polygon, etc.) are inheritable. These syntaxes are used to construct below GeoBI context ontologies.

\section{OWL-Based Top-Level Mobile GeoBI Context Ontology}

For the purpose of enabling context reasoning, the top-level UML-based context model (Figure 1) has been translated into OWL-based GeoBI context ontology (Figure 5). Note that colors (red, blue) are used by CmapTools to highlight some categories of links, and sometimes by us (other colors) to differentiate intersecting links. They do not carry a particular meaning. 
Table 1. Some syntactic equivalence between OWL and CmapTools.

\begin{tabular}{|c|c|c|}
\hline & Owl/RDF/RDFS Syntax & CmapTools COE labels \\
\hline \multirow{4}{*}{ Axioms } & rdfs: subClassOf & Class A “are” class B \\
\hline & $<$ A rdf: about $=$ “\#obj”/> & The object < obj > "is a" member of the Class A \\
\hline & owl: equivalentClass & Class A “same as" class B \\
\hline & owl: disjointWith & Class A “cannot be” class B \\
\hline \multirow{4}{*}{ Descriptions } & owl: oneOf & Class A “is defined as one of” individual B \\
\hline & owl: intersectionOf & Class A “is defined as all of” classes B, C, etc. \\
\hline & owl: unionOf & Class A “is defined as any of" classes B, C, etc. \\
\hline & owl: complementOf & Class A “exact opposite of” class B \\
\hline \multirow{3}{*}{ Cardinalities } & owl: minCardinality & Class A “at least $<\mathbf{N}>$ ” individual $B$ \\
\hline & owl: maxCardinality & Class A “at most $<\mathbf{N}>$ ” individual B \\
\hline & owl: cardinality & Class A “exactly $<\mathbf{N}>$ ” individual B \\
\hline
\end{tabular}

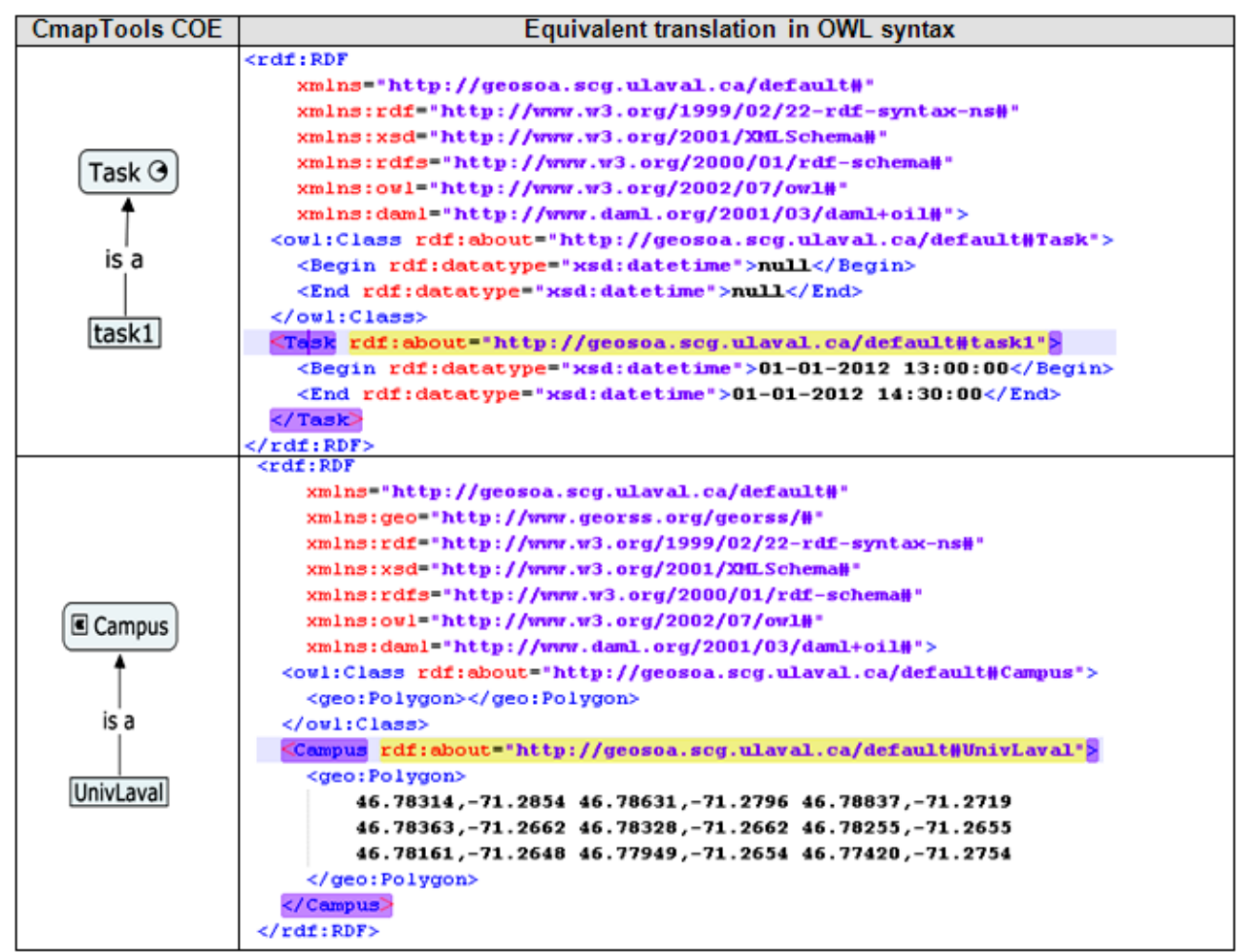

Figure 3. Integration of temporal and geospatial pictograms and properties into CmapTools COE and OWL.

That top-level context ontology provides a generic overview of a mobile GeoBI context stakeholders, highlights its hierarchical multilevel structure, and defines it as a union of (i.e. "any of") three hierarchical context levels (personal, ambient, and surrounding contexts) which are in turn defined as combinations of context dimensions.

The union relation is more explicit in the OWL excerpt provided in Figure 4. The context dimensions were selected by [5] based on their relevance for mobility and BI activities (See Table 2) and were dispatched into the three hierarchical context levels to match with the human mental representation of space identified by [17]: 1) 


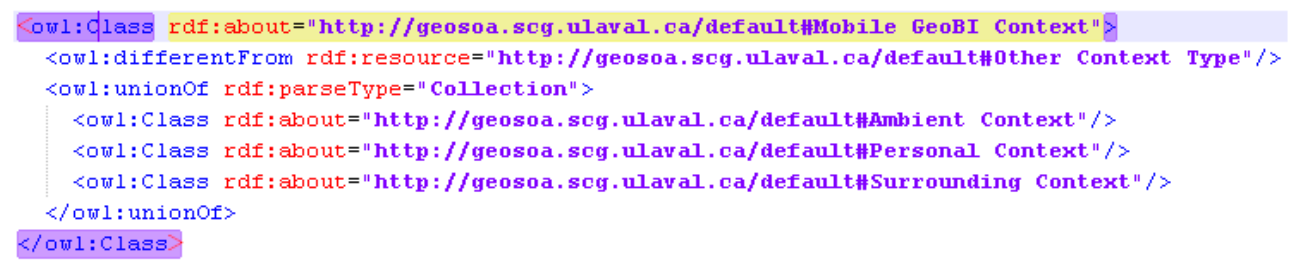

Figure 4. Excerpt of Owl defining mobile GeoBI context as a union of personal, ambient and surrounding contexts.

the body space, 2) the space around the body, and 3) the navigation space. The personal context is about information regarding the user such as his profiles, goals, tasks, etc. The ambient context is about the immediate environment around the user in which things can be more accurately sensed by the user, while the surrounding context potentially covers the whole surrounding environment. The ambient context is a subset of the surrounding context. Their difference resides in their scope. Indeed, ambient context is a restriction of surrounding context to a given area of Interest (AOI), which leads to a kind of projection (limitation) of the dimensions content inside this AOI. Conversely, a surrounding context is somehow, an extension of ambient context to a wider AOI. Both have the same context dimensions.

As nobody is completely cut off from the world, especially in nowadays global business world, this context ontology provides a suitable way to tackle the issue of context-sharing, i.e. the fact that a local business contextual information (e.g. gas price in Quebec City) may be affected by a remote context whatever the business context (e.g. wall street stock exchange) or any other context (e.g. war in Libya) is. This is expressed in the proposed ontology (Figure 4, Figure 5) by the relations connecting local and remote contexts through the shared contextual information, and is exemplified by the following individuals: 1) “Gas business in Quebec city” which is an instance of "Local Context”; 2) "War in Libya” which is an instance of "Remote Context”; 3) "Daily oil production down" which is an example of "contextual info" shared by the remote context and that may influence the local context. In the figure, the couple of relations shares 1/influences 1 and shares 2/influences 2 exposes the fact that a remote context may share (share 1) a contextual information that influences (influences 1 ) the local context, and conversely, the local context may may share (share 1) a contextual information that influences (influences 1) a remote context. To describe the spatial coverage/attribute of an object instance (e.g. gas Business in Quebec City) the has Geom relation is used to list its geometric coordinates in Geo-OWL format.

Details composing personal, business, technological, spatial, social, and environmental contexts are inventoried and designed into detailed ontologies in Section 4.

\section{Low-Level and Detailed Mobile GeoBI Context Ontologies}

Besides top-level concepts, more specific concepts are required to enable mobile GeoBI applications sense or retrieve detailed contextual information (e.g. user position, his office address). This section provides for each context dimension (personal, business, technological, spatial, social, and environmental contexts), a detailed context ontology.

\subsection{Personal Context Ontology}

The personal context retrieves any relevant contextual information regarding the user. The well-known FOAF (friend of friend) ontology already allows describing persons and their relationships [18], but is mainly dedicated to describing social networks rather than GeoBI contextual information. For instance, information about a person's agenda, tasks, or preferences is absent in foaf. A GeoBI personal context ontology is therefore required.

Table 3 shows key concepts identified as relevant and Figure 6 presents the proposed ontology. It defines a GeoBI personal context as an aggregation of (i.e. union of) the person's identity, cognition, tasks and goals and encompasses the foaf: person entity instead of creating a new one. This enriches the proposed ontology with the so popular FOAF ontology.

The personal context dimensions are intended to bring answers to questions regarding the mobile person, precisely:

1) "Who is he?" i.e. his identity. The notion of identity has been organized into simplified and consistent profiles which highlight the person’s psychological, social, professional, physiological characteristics. In addition, a 
Table 2. Relevant dimensions for a mobile GeoBI context.

\begin{tabular}{cccc}
\hline $\begin{array}{c}\text { Context } \\
\text { Level }\end{array}$ & Dimensions & Description & Relevance for \\
\cline { 2 - 3 } & & Mobility & BI \\
\hline
\end{tabular}

\section{$++$}

The mobile person's goal, agenda,

Goal needs, intentions or interests in what he is doing or intend to do.

Identity The person's role and identity such as his civil, professional or use profile.

Personal

context In short, the person's psychological profile (mood, behavior) and preferences (like/dislike).

Tasks carried out. These can be Mobility tasks (e.g. driving, walking, etc.), BI tasks (requesting decisional data, meetings), Communication tasks (calling, messaging, etc.), Other tasks (e.g. carrying a weight, painting a wall, etc.)

$+$

$++$

$++$

Pertinent to assist the user to reach the expected task. For example, detecting the user's intention to visit the closest client by sensing his current position and direction, and then propose him the most rapid route and display the most up to date and critical indicators about this client.

Moderately relevant for accessing spatial navigation support applications.

$++$

Strongly relevant for supplying right information to the right decision maker based on his identity.

Relevant to know for example where the user should not go (e.g. agoraphobic or claustrophobic), or would like to visit.

$++$

Relevant for handling in which ways the mobile worker might be "advised" and assisted in his activities.

Relevant for Mobility tasks

$++$

Relevant for $\boldsymbol{B I}$ tasks

$++$

Of course, highly

relevant for business

intelligence support

Not really relevant for mobility

$++$

$++$

Relevant for way finding Relevant for accessing and support or delivering context-aware services processing context-based business data

$+$

Social context is not only about social networks,

Surrounding context

Social but also about culture, power systems (i.e. politics) and resources management (economy). In short, it's about social organizations of humans and resources.

Moderately relevant for asking location information or discovering POIs of a society

$++$

Strongly pertinent for understanding and dealing with social groups, local culture, resources and institutions.

$++$

Strongly relevant for adapting activities (Mobility and BI tasks) to environmental conditions and available services.

$++$

Strongly relevant for spatial navigation
$++$

Strongly relevant for locating companies, customers and for delivery issues (costs, delays, etc).

All contexts
and $\begin{gathered}\text { Tempo-ral } \\ \text { dimensions } \\ \text { above }\end{gathered}$

Refers to the time or the period during which tasks are carried out, events occur, resources are available, etc. For us, the temporal dimension is specific and affects all context and dimensions we treated above. This specificity is explained in [5].

\section{$++$}

Relevant for journey duration, transportation means availability time, etc.
$++$

Pertinent for monitoring business evolution over time, timeliness, etc. 


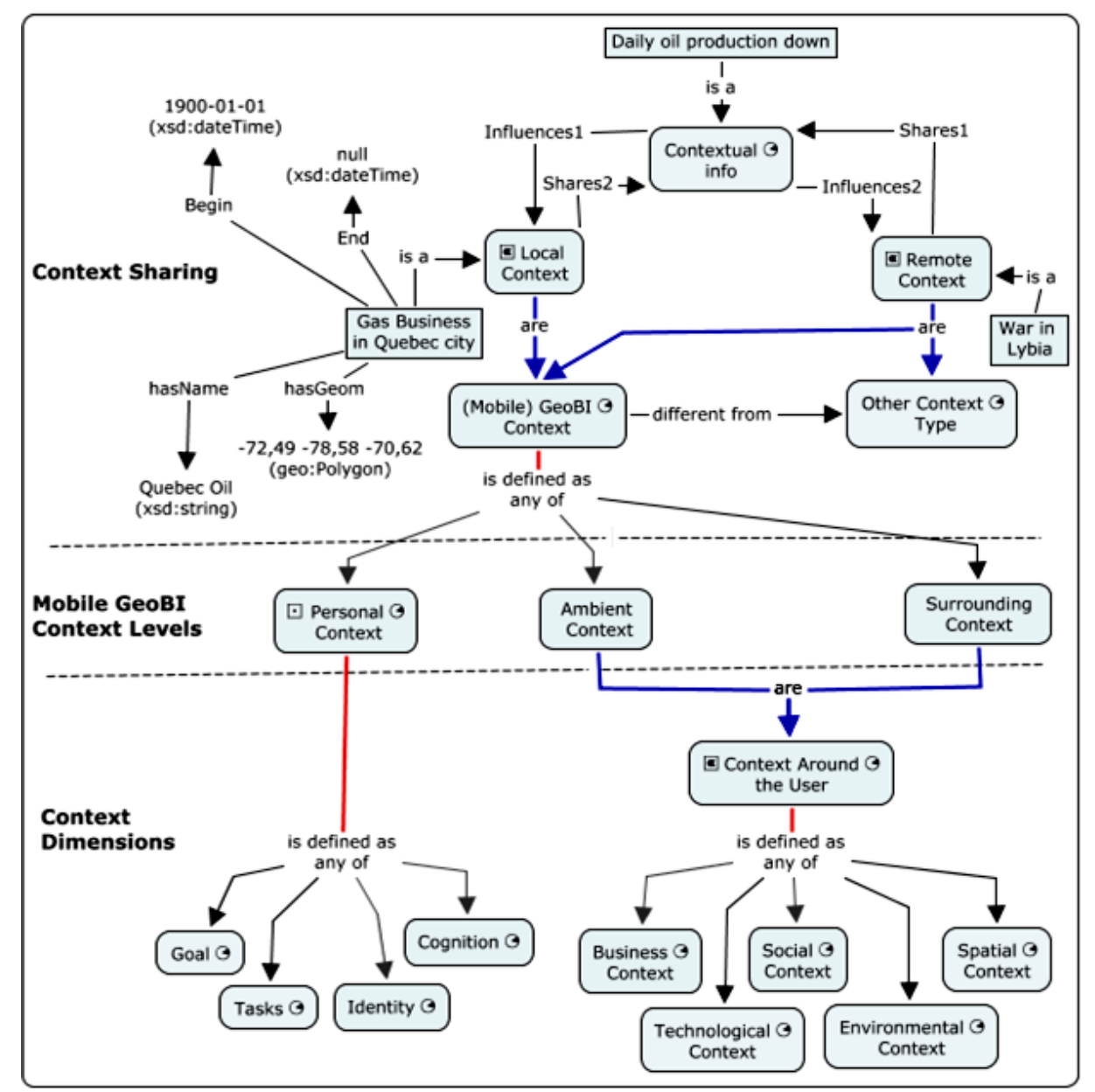

Figure 5. Top-level multilevel ontology for Mobile GeoBI contexts.

mobile user may have several use (or user) profiles (e.g. facebook, twitter, linkedIn). Modeling this way allows linking a profile to another one. For instance, some user profiles (e.g. facebook, twitter) may be linked to the user's social profile, while his linkedIn account could be linked to his professional profile.

2) "What does he need or intend to do?" i.e. his goals. Generally goals are related to things that could motivate or explain the user's actions such as his intentions (e.g. get promoted), needs (e.g. sales training), interests (prospecting customers) or his professional missions, functions or roles. Intentions, needs and interests have been separated from business objectives to distinguish personal goals from corporate goals.

3) "How does he think, act, react or feel things?" i.e. his cognition. The user's cognition aspects were simplified here to focus on what might be relevant for context-awareness such as preferences and psychological profile: e.g. hobby, mood, temperament, personality traits. For instance, knowing a person is nervous may help manage and anticipate his behavior in a given context (e.g. contract negotiation). As personality traits, the big five personality traits (openness, consciousness, etc.) defined by [19] are used. These cognition elements can be for example, 1) manually collected and input from personality tests and measures [20], or 2) automatically captured by using various sensors (e.g. camera, microphone, GPS, accelerometer, cookies, activity logs, etc.) to assess the user's mood [21], personality traits [22], etc.

4) "What is he doing/what does he have to do?" i.e. his tasks which can be separated into two main groups: private tasks (e.g. eating, sleeping, leisure activities) and organization tasks. This categorization provides the possibility to define priorities between tasks. For example, cancelling or rescheduling a private task instead of any other organization task when a business emergency occurs.

Since it is about the mobile person, the personal context is location-based and time-dependent and may be used to locate the person or characterize his deeds and state at a given place (e.g. watching TV at hotel while 
Table 3. Relevant personal contextual information for a mobile GeoBI context.

\begin{tabular}{|c|c|c|}
\hline Context Level & Context Dimensions & Relevant contextual information (Key elements and details) \\
\hline \multirow{14}{*}{$\begin{array}{c}\text { Personal } \\
\text { context }\end{array}$} & \multirow{5}{*}{ Identity } & Civil Profile (Name, Address, Marital Status, Languages, etc.) \\
\hline & & Professional Profile (Diploma, Skills, Experience, Performance, Role, Results, etc.) \\
\hline & & $\begin{array}{l}\text { Physiological Profile (Body Description [Weight, Height, Skin Color, Hair color, Eye Color]; } \\
\text { Body Disabilities [motor, visual, hearing, etc.]; Genetics [DNA, Blood Group, Rhesus, } \\
\text { Fingerprint, etc.]; Health [diseases, allergies, etc.]) }\end{array}$ \\
\hline & & Social Profile (Family, Community, Association, Friends, Relatives, Life Style, etc.) \\
\hline & & Use(r) Profile (Login, Password, Rights, etc.) \\
\hline & \multirow{3}{*}{ Goal } & $\begin{array}{l}\text { Needs/Intentions (Business needs to fulfill or objectives to reach at personal level. E.g. } \\
\text { Need to register } 10 \text { new customers today!) }\end{array}$ \\
\hline & & $\begin{array}{l}\text { Interest (Business centers of interest. E.g. selling innovative products, prospecting customers, } \\
\text { analyzing revenues from delivery services, keeping informed of stocks level in the company's } \\
\text { closest shops, etc.) }\end{array}$ \\
\hline & & Agenda (Planned interest or tasks. E.g. from 9 to 10 a.m. prospecting customers) \\
\hline & \multirow[b]{2}{*}{ Cognition } & Psychological Profile (Temperament, Mood, Hobby, Personality Traits, etc.) \\
\hline & & $\begin{array}{l}\text { Preferences (Indicate what the decision maker likes the most for each of his profile. E.g. } \\
\text { Civil Profile: A perfect bilingual, but prefers speaking French, etc.) }\end{array}$ \\
\hline & \multirow{4}{*}{ Tasks } & $\begin{array}{l}\text { BI Tasks (Type [consulting/requesting decisional data], concerned metrics, } \\
\text { Qty of Data to download, costs, etc.) }\end{array}$ \\
\hline & & Mobility Tasks (Type [walking, driving, etc], Duration, etc.) \\
\hline & & $\begin{array}{l}\text { Communication Tasks (Type [e.g. calling, messaging, chatting, twitting, etc.]; Duration, } \\
\text { Network, Costs, etc.) }\end{array}$ \\
\hline & & Physical Tasks (Duration, Difficulty, Distance, etc.) E.g. carrying a box of products, etc. \\
\hline
\end{tabular}

accessing BI data). In addition, thanks to symmetric (symbol "<=>”) relations "is linked to", it might be reasoned and deduced that 1) goals and cognition are linked, 2) cognition and tasks are linked, or that 3) goals and tasks are linked, etc. Figure 7 shows an excerpt of OWL showing these symmetric relations between preferences and profiles, and between profiles and goals.

\subsection{Surrounding Context Ontology}

\subsubsection{Business Context Ontology}

For a mobile professional, getting informed of local business actors, mainly organisations retrieving contextual information about their activities, their products, available resources and existing markets, can be crucial to assess his local business context and conduct convenient location-based business analysis (e.g. analysis of spatial distribution and catchment areas of competitors/clients, etc.). Business here is considered as " $a$ collection of activities carried on for whatever purpose, be it science, technology, commerce, industry, law, government, defense, et cetera." [23].

The business context ontology model proposed in Figure 8 is built upon these key concepts retrieved from the literature as relevant dimensions for business context: Organization [24]-[26], Activity [24] [25], Resource [24] [27], Market [24] [25], and Strategy [24] [25] [28]. Business context is defined as a set (union property in OWL) of organizations having activities following a certain strategy to produce or use resources and make them available on markets. These key concepts are described below.

Organization [24]-[26]: Organizations include common associations, administrations and business organizations. They are potentially business actors (persons too) that may be engaged in business relations (e.g. competition, partnership). They are generally organized into management entities (e.g. department, services, teams, etc.) leaded at different levels by executives, managers, foremen, etc., who are accountable among human resources (e.g. employees, consultants, etc.). 


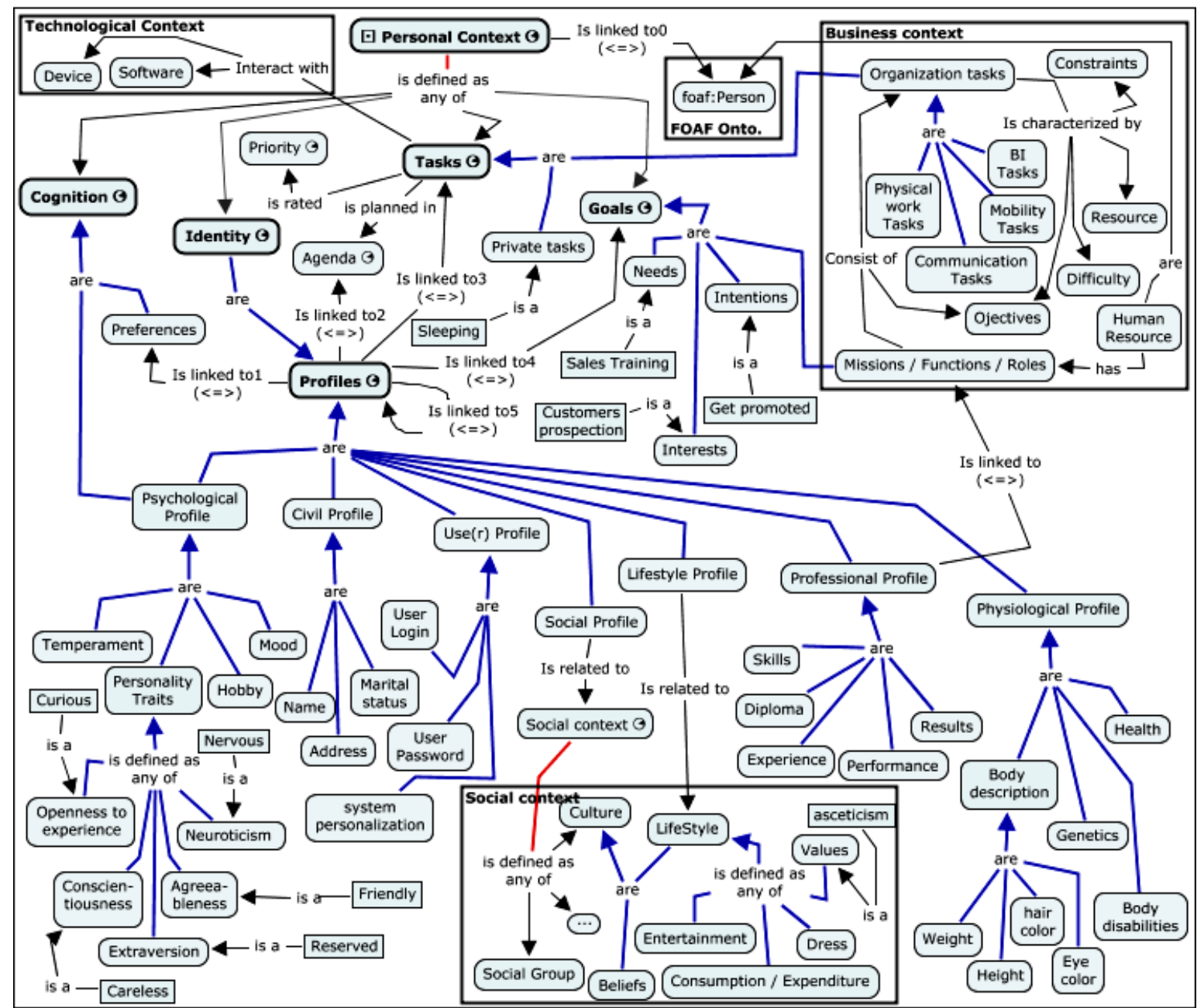

Figure 6. Detailed personal context ontology.

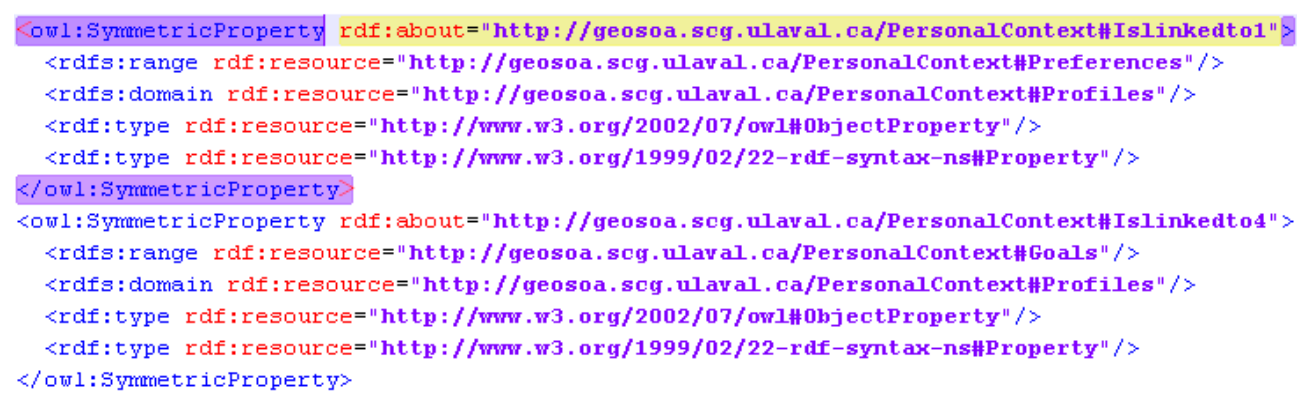

Figure 7. Excerpt of OWL illustrating symmetric relations between preferences and profiles, and between profiles and goals.

Activity [24] [25]: Activities (e.g. extracting oil) can be divided into processes (e.g. distribution) which can be in turn sequenced into tasks (e.g. extracting). These tasks are designated as "organization tasks" to differentiate them from private tasks. In our point of view, they can be classified into: 1) Business tasks (e.g. selling), 2) BI tasks (e.g. analyzing sales), 3) Mobility tasks (e.g. delivering), 4) Communication tasks (e.g. advertising), and 5) Physical work tasks (e.g. carrying a box).

Resource [24] [27]: Resources are used in activities to make products. Instead of limiting resources to goods and services, the ontology also includes Human and Financial resources. Products are also resources even if they 


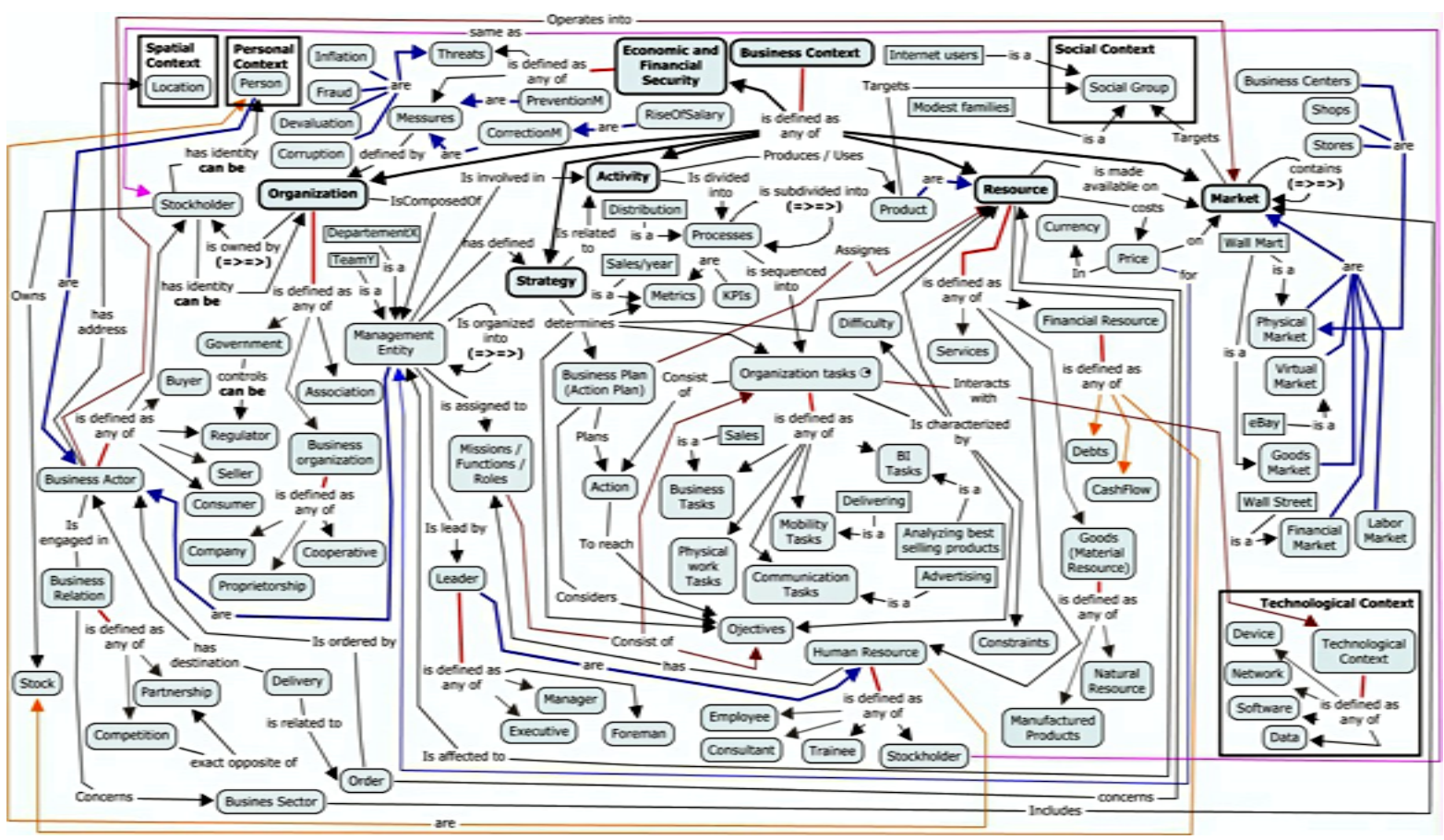

Figure 8. Detailed business context ontology.

are the result of transforming other resources. Similarly, stockholders are human resources since they bring funds, ideas, and opportunities to their organizations.

Market [24] [25]: Resources are generally made available on markets where business actors (sellers, consumers, regulators) can operate to sell, buy, or regulate (regulators, e.g. the government) them. Markets can be grouped, not only, into: 1) physical markets (e.g. wall mart), 2) virtual markets (e.g. eBay), 3) goods markets (e.g. wall mart), 4) financial markets (e.g. wall street), 5) labor markets where businesses hire human resources (e.g. employment agencies), etc.

Strategy [24] [25] [28]: "Corporate strategy is the pattern of decisions in a company that determines and reveals its objectives, purposes or goals, produces the principal policies and plans for achieving those goals, and defines the range of business the company is to pursue..." [29]. The notion of strategy relates then to the organization vision and politics. In our point of view, it can be concretely derived from strategic questions regarding the organization vision, such as, but not necessary exhaustive ones: 1) Objectives to reach 2) Tasks to implement 3) Required resources to mobilize 4) Business plan or actions-plan that defines actions to take, resources to use, deadlines to respect (Actions begin and end), and assigns missions to involved actors (management entities and human resources); 5) effective decision support tools [29], e.g. Metrics and KPIs (Key Performance Indicators), to measure and monitor business performance and react accordingly by taking suitable decisions. Figure 9 exposes an OWL excerpt of the strategy ontology.

\subsubsection{Technological Context Ontology}

Retrieving technological contextual information is crucial for at least the following reasons:

- 1) BI as well as context-aware applications strongly need and use technologies to retrieve (from data sources) or capture (from sensors) the right information and deliver it (via networks) to the right person at the right time (thanks to convenient software) for the expected use (through convenient Human-Computer Interactions) and in consideration of the information security (privacy, confidentiality, etc.).

- 2) Organizations' tasks heavily rely on IT (information technologies) so that events occurring in the technological context (system failures, software bugs, data spy, etc.) may affect the way business is conducted and its results (e.g. customers' complaints, etc.).

- 3) Given that mobile devices (e.g. smart phones) cannot capture surrounding information beyond a given distance, the more a context-aware mobile GeoBI application knows about his technological context, the bet- 


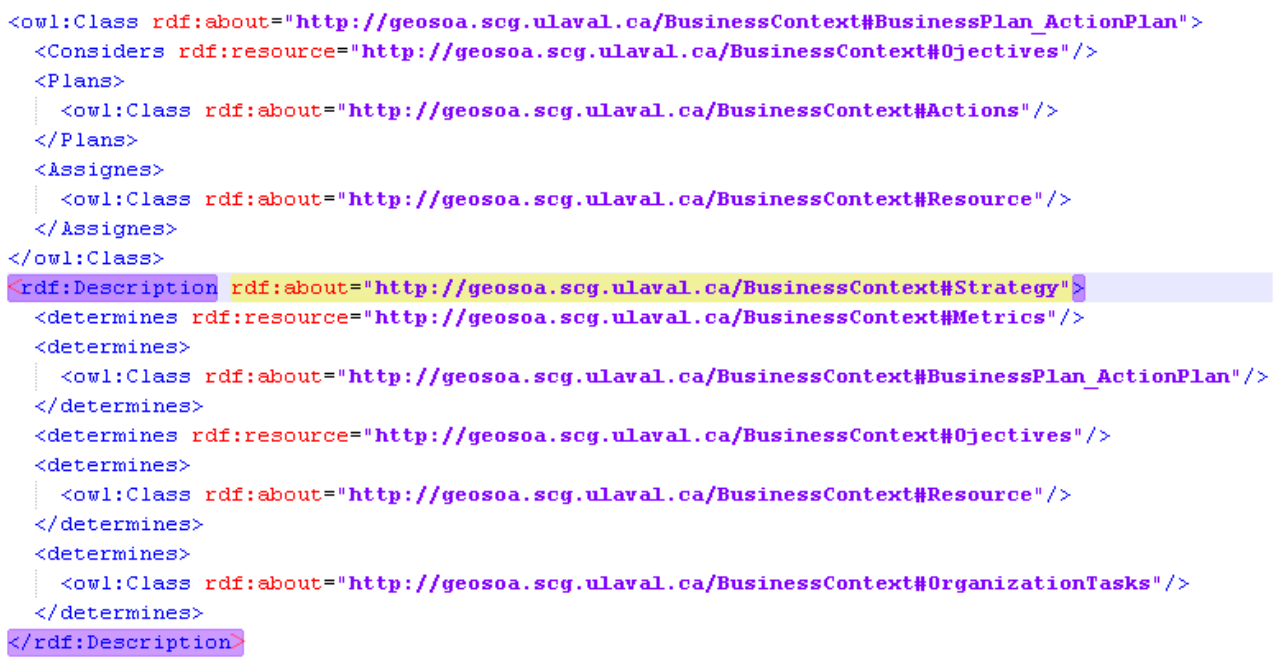

Figure 9. Excerpt of OWL defining the strategy ontology.

ter it can manage to adapt its operations. For instance, to avoid network issues (e.g. disruptions) to a mobile decision maker moving from Address A to address B throughout the city while requesting BI data from his organization, a context-aware mobile GeoBI application, by accessing the city's technological context map (e.g. via context-providers/web services), could propose, by invoking a location-based service, a technologybased route that avoids as much as possible, 1) areas not covered by compatible networks or where networks are off (e.g. because of last night storm), 2) underground ways unless they have internal network relays, etc.

Figure 10 provides a technological context ontology to help handle these crucial needs. It identifies and describes a technological context as a combination of at least five technological key concepts:

Hardware: regroups networks and devices. Computers, mobile phones as well as sensors are considered as geo-localizable devices having some characteristics which may be compliant with other devices or with some networks. Networks can be wired or unwired, are localizable through their catchment areas, and have characteristics like IDs, carriers, protocols, bandwidth, etc.

Software: is classified into 1) operating systems installed on a hardware to manage it, 2) system-oriented software (e.g. WinLogon, web services, etc.) dedicated to be used by another software, 3) and user-oriented software end-users can interact with (e.g. Word processing).

Data: is about available data repositories and sources including data files, databases, and data warehouses. They can be described by data models, metadata, and their content quality (relevance, accuracy, integrity, etc.) to let applications accessing them know about their data structure, and level of confidence.

HCI: Human Computer Interactions refers here to the available ways (interfaces) a user can choose to interact with a hardware or software, according to his needs, physical capabilities or occupation. For example, a mobile professional who is driving will foster sound interactions rather than only displaying texts. The ontology also allows defining which Computer interface and Human interface should be combined to perform an interaction. For instance, it can be defined that a speech input interaction is achieved by using a microphone (computer interface) and voice (human interface). The context-aware GeoBI application will then be in charge of detecting the user's needs, physical capabilities, and occupation to propose the right interaction mode to use.

IT Security: is about the information security regarding hardware, software and data in a given area. It involves information about IT security level (e.g. Privacy), information access control (e.g. encryption) and potential security threats (e.g. hackers). Since security is a sensible matter, not any information regarding security should be communicated to any user requesting it. Furthermore, security information targeted here is just about getting the user informed of security risks or issues when accessing a network, a website, a data repository, etc. For example, is the available WIFI access point a fake WIFI hotspot [30] or a secure one? What is the security rating of the website the user is willing to access? etc.

\subsubsection{Spatial Context Ontology}

Capturing and reasoning on the spatial context surrounding mobile professionals is crucial to provide a location- 


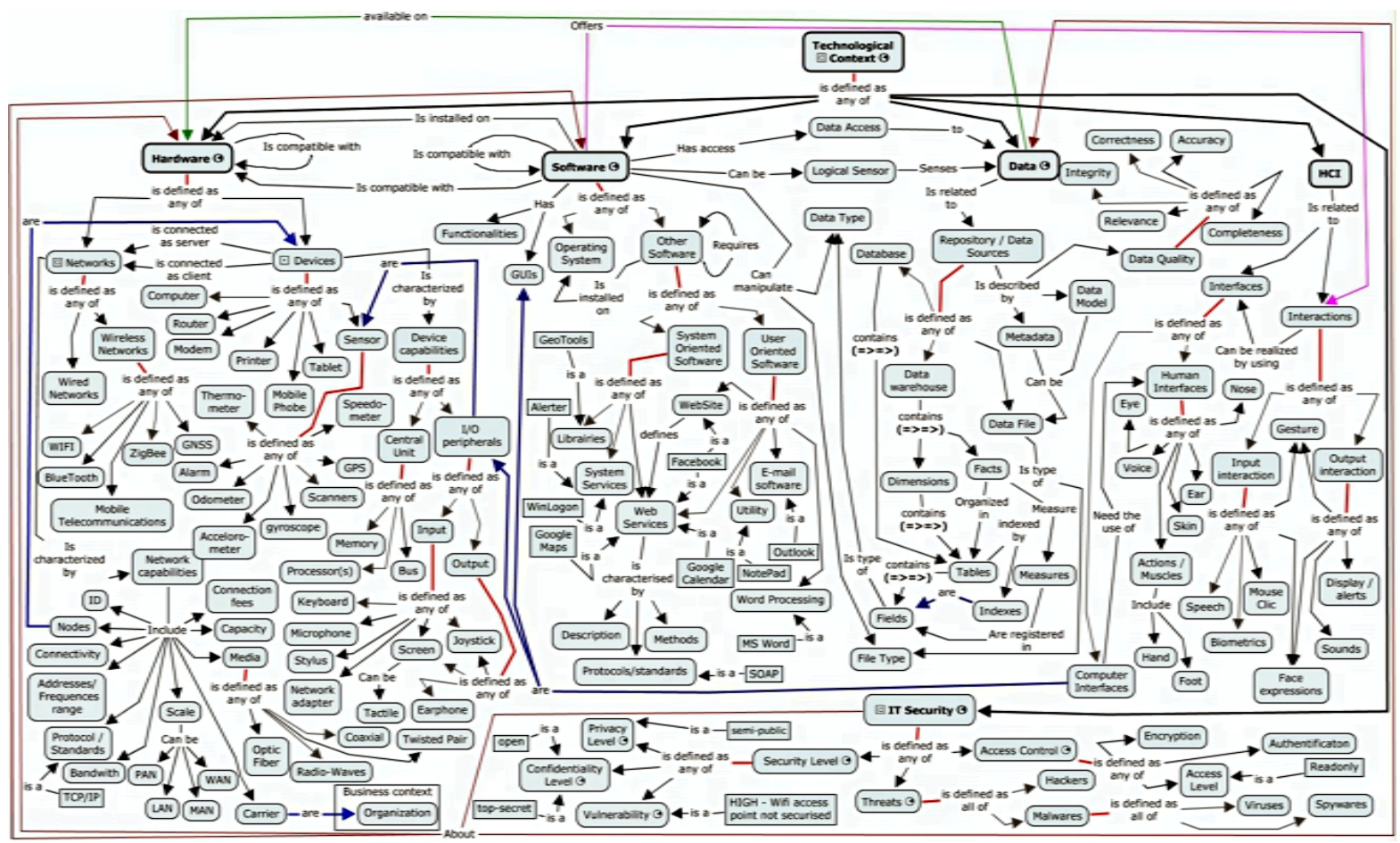

Figure 10. Detailed technological context ontology.

based and context-based mobile GeoBI decision support.

Based on [31]-[33], we define Spatial context as the locations and spatial relations describing relevant physical features including living beings (people, animals), physical geography features (e.g. lands, vegetation), human-made features (e.g. territories), and Points of Interest (POIs). The user's spatial navigation context (e.g. current location, orientation, etc.) and the location security are also relevant key concepts in characterizing the user spatial context. Figure 11 provides a spatial context ontology detailing the semantics of these key concepts. Their relevance is highlighted below.

Living beings: Besides localizing people (e.g. crowd, clients) as usual, the ontology also allows to localize animals since certain business activities might concern animals (e.g. livestock farming). Moreover, locating the presence of herds may be helpful for traffic purpose or safety issues (e.g. Alert of presence of wolves).

GeoObjects: This is a generic concept referring to geo-localizable objects and used to make difference from living beings. It is a super class of physical geography and human-made features.

Physical geography features: This concept is intended to capture or retrieve physical geography characteristics which may be relevant for business professionals, such as: lands (e.g. mountains, plain, etc.), vegetation, water, soil (e.g. sand), subsoil (e.g. oil, gold, silver), etc. This is undeniably relevant for governments, mining, natural resources management companies, etc.

Human-made features: are about geo-spatial objects built by human beings. Two main relevant groups are proposed:

- Territories: spaces delimited by human beings to distinguish a place from another one for legal (e.g. country borders), administrative (e.g. district), or social (e.g. friend room) purposes, etc. They span from wide (e.g. countries) to small spaces (e.g. rooms).

- Infrastructures: structures and facilities constructed for a given usage, like: 1) Housing, hospitality and leisure infrastructures, 2) Health infrastructures, 3) Education infra-structures, 4) Transportation infrastructures (rails, vehicles), 5) Water infrastructures, etc.

Location: Whereas living beings and geoObjects are semantic geo-spatial entities, location is about positioning them absolutely or relatively to another location. For the purpose of location-based reasoning and in accordance with Geo-OWL syntax [16], we have designed a GeoRSS GML-based ontology which defines absolute, relative and indirect location (see round-corner rectangle in Figure 11). 


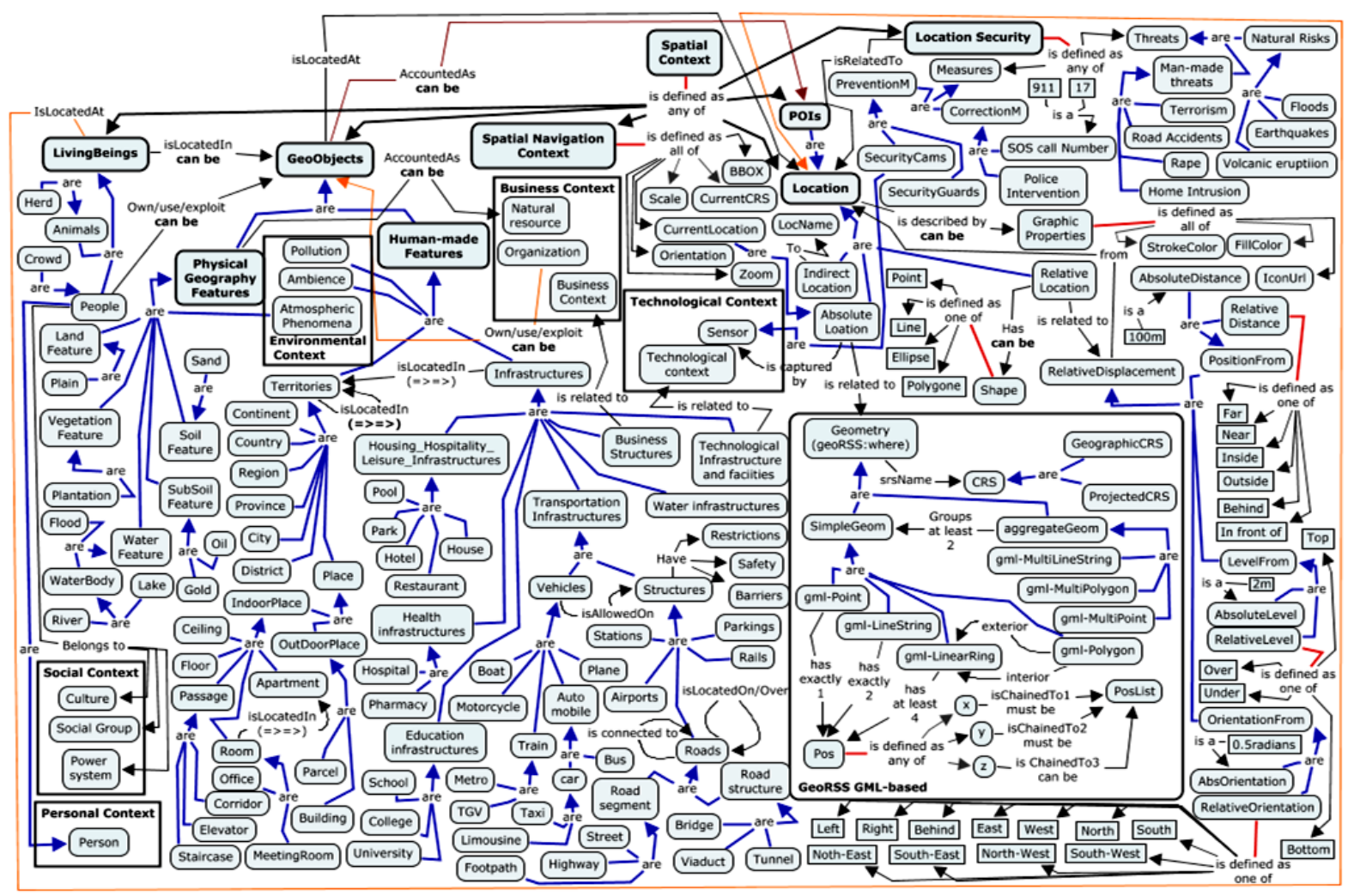

Figure 11. Detailed spatial context ontology.

An absolute location is a series of absolute coordinates in a given spatial representation system (SRS), forming a well-known geometry (point, line, polygon, multi points, etc.).

A relative location is defined relatively to another location by a relative displacement. The final location that is referred to must be absolute. The user's position is the default absolute location to be referred to when another one is not indicated. A relative displacement is a super class of:

- Position displacement (Position From): absolute (e.g. 100 meters) and/or relative position (e.g. far, near, inside, behind, etc.) from the referred location (e.g. $100 \mathrm{~m}$ near Laval)

- Level displacement (Level From): absolute (e.g. 2 m) and/or relative level (e.g. under, over, top, etc.) from the referred location (e.g. the mobile phone is $2 \mathrm{~m}$ over, i.e. above, the soil)

- Orientation displacement (Orientation From): absolute and/or relative orientation (left, right, east, etc.) from the referred location (e.g. the mobile phone of 0.5 radians towards east).

Even though a relative location does not have a well-known geometry, it can be represented by an approximate shape (point, line, polygon, ellipse, etc.) which describes its appearance.

Instead of locating them absolutely or relatively, living beings and geoObjects may also be simply localized by a place name (e.g. Wall Street is located in New-York) whose coordinates may then be fetched from online gazetteers [34]. This way of localization can be considered as indirect location since one more step is needed to get a more precise location.

POIs: "A point of interest is a geographical entity, such as a landmark, business, school, or public building. They include such places as airports, university campuses, and parks...” [35]. POIs can be marked as interesting by the mobile professional himself, users sharing their experience from social networks or by organizations (e.g. government, map vendors, etc.). Since POIs are generally places often visited by people, mostly tourists, a mobile professional might want to analyze sales performance (e.g. best-selling products, best-selling periods, etc.) realized in such places. A mobile business professional may also mark business entities (e.g. shops, competing companies, banks, etc.) as POIs in order to retrieve their location faster when needed.

Location security: "The issue of security, whether it be overall hotel security or more specifically room or car park, security has been judged to be an important issue" [36]. [37] and [38] also reports that security is 
among the most important criteria for business travelers' accommodation selection. Moreover, since mobile people are evolving in dynamic, unfamiliar, and unpredictable areas [39], they should be informed of potential security threats (e.g. terrorism, earthquakes) and security measures (e.g. SOS call number).

Spatial navigation context: To provide a mobile professional with a location-based decision support (e.g. "Which are the fast selling products versus the best profitable products per store within $5 \mathrm{~km}$ of my current location during the last two months?"), his spatial navigation context should be known (e.g. current position, orientation, BBOX, SRS, etc.).

\subsubsection{Social Context Ontology}

Social context is often reduced to social network relationships [40] [41]. But, since Business Intelligence is concerned, it should be extended to consider the organization of society given that what mainly interest business people when they arrive somewhere is in short, “can I do business here and how?” Our response is, by taking into account the social groups composing the society, its powers (e.g. political, economic, judiciary, religious, etc.), system of power (e.g. democracy, theocracy, dictatorship, etc.), institutions, culture (language, beliefs, lifestyle, etc.), and rules (e.g. norms, legislation, etc.). Some works [42] and [43] also support that social context includes culture and politics: "Social context includes the culture, distribution of power, and the social norms, habits, practices, expectations and preferences held by a group regarding its present and past interaction" [42]. Moreover, [44] underlines that "Culture becomes one of the most critical make-or-break factors in successful multinational corporate operations." Thereby, such social contextual information provides richer parameters for conducting location-based social analysis like: In the current sales zone, where are located Chinese customers that purchase at least $\$ 100$ of French cheese each month? Figure 12 shows the proposed Social context ontology built upon the key social concepts highlighted above. The Power concept is for example decribed in that ontology as possibly having various declinaisons such as political, economic, judiciairy or religious powers, etc., that may adopt a given system of ruling (e.g. democraty, theocraty, etc.).

\subsubsection{Environmental Context Ontology}

Since Kyoto protocol in 1997 on reducing greenhouse gas emissions, organizations have to be aware of environmental issues and restrictions enacted by national and international laws. To meet these greening business measures and avoid penalties several governments and companies have even integrated the notions of sustainable development and environment protection into their strategies and business management processes, from products manufacturing to distribution activities [45].

To keep mobile professionals aware of environmental challenges, the environmental context ontology in Figure 13 is proposed to help handle environmental conditions and issues regarding:

1) the surrounding ambience, e.g. Noise, indoor, outdoor ambience, etc. [46].

2) the atmospheric conditions, e.g. climate, seasons, weather, etc. [47] [48].

3) the biodiversity, e.g. endangered species, ecosystems [49].

4) the environmental security i.e. environmental threats (e.g. pollution, erosion, etc.) and protection measures (e.g. waste recycling, pollutions bonds, etc.) regarding the visited location [50].

The ontology has been limited to some relevant contextual information that might interest business people. Therefore, it might be extended with other information.

\section{Context Acquisition and Quality}

Contextual information identified in the different context ontologies afore proposed can be collected in real-time, from physical sensors sensing physical world (e.g. location, motion), and logical sensors sensing IT activity (e.g. detecting the user's location from his IP address). But some contextual information may require a manual input (e.g. the user's personality traits, etc.). This means that at a given instant t, not all contextual information is acquired and fed in the context ontology: there could be missing one. Moreover, it might be unknown, ambiguous, imprecise or erroneous [51]. So, context-aware mobile applications exploiting GeoBI context ontologies must be able to deal with incomplete and uncertain contextual data.

Context uncertainty modelling and reasoning are still important research issues [52]-[54], that cannot be completely covered in our current research thesis whose focus regarding context is mainly identifying and modeling contextual information that is relevant for mobile Business Intelligence. Issues regarding data security, data confidence and data certainty, etc., will be addressed in further work. 


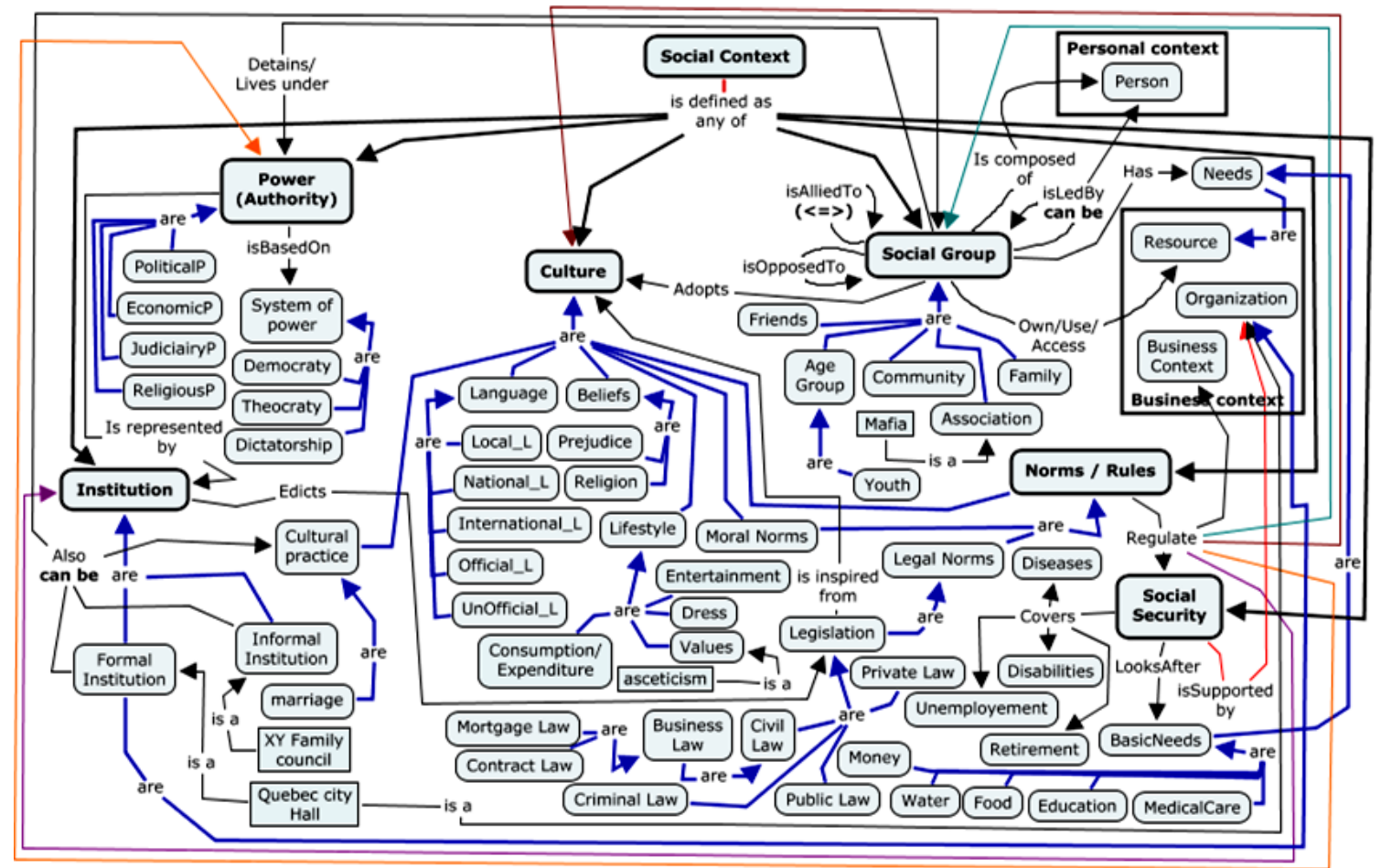

Figure 12. Detailed social context ontology.

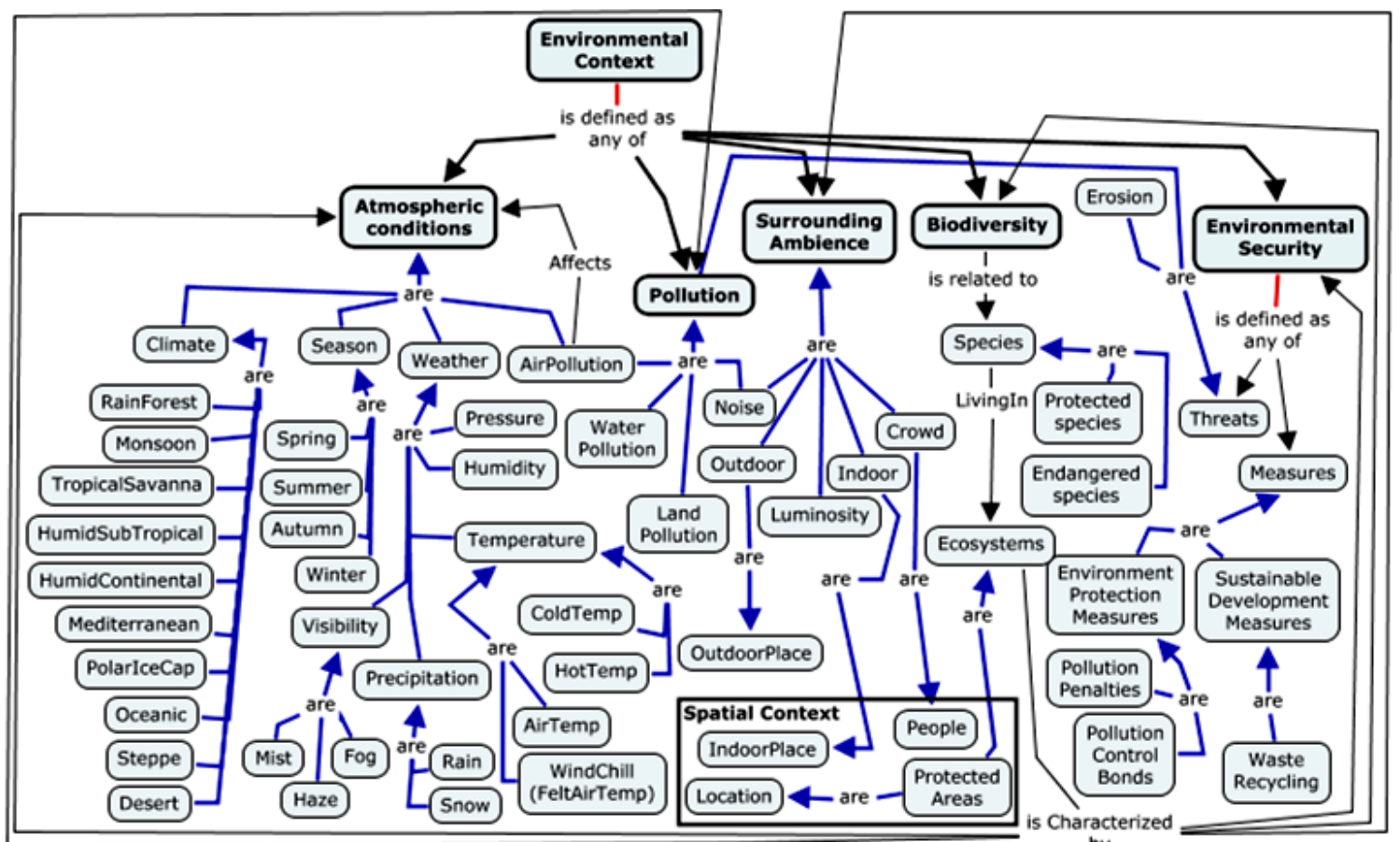

by

Figure 13. Detailed environmental context ontology.

However, in our current model, context uncertainty is already handled at two levels of quality control: 1) by defining quality parameters and metrics for sensors [55] and/or 2) by associating/annotating contextual entities with Quality of Context metrics [54] such as certainty level [51]. The first level is defined in the technological context ontology (Figure 10) by modeling quality properties for sensors (devices or software) used to capture 
the information. Hence, to the GPS sensor installed on the mobile used by salesman Steve, could be attached quality metrics regarding its precision (e.g. $20 \mathrm{~m}$ ) and accuracy (e.g. 70\%) parameters in measuring the user's current position. Then, the accuracy and precision of the captured value can be derived from the GPS device quality parameters. The second level of quality control consists of annotating contextual entities with Quality of Context metrics QoC [54], by indicating for example their level of certainty. For example, the user's position previously captured might be corrected based on the GPS precision and stored as being $90 \%$ sure, whereas a user profile information input (e.g. work experience) might be moderated by administrators and set as doubtful (e.g. 0.3). The certainty value might be an estimation or a probability [52] [56]. This second level is not explicitly modeled because of readability issue, but thanks to OWL dynamic capability for creating additional properties, a certainty label and value can be created at runtime. Unknown or missing information is considered as null and the user may be requested to enter it if required. For each contextual information, its data type is annotated to allow reasoning engines make the difference between text, numeric, and geospatial, etc. values.

Context uncertainty also includes geospatial and temporal dimensions of contextual information. Thanks to our integration of these spatial and temporal dimensions in our model, this issue is half solved, at least regarding data freshness and location resolution since we can know the validity time of a contextual information (begin and end time) and its precise location thanks to its geographic coordinates (in a given) our model retrieves as geometric object for geospatial features. However, this can be enforced by the use of QoC metrics such as timescale units (e.g. seconds, hours, months, years), location resolution (e.g. district level, city level, state level, etc.).

Further enchancement could be brought to our current solution of handling context uncertainty based on QoC annotation [54], by considering the use of:

- fuzzy logic [57];

- probabilistic logic [56];

- Markov models [58];

- Bayesian networks [59];

- Dempster-Shafer theory [60].

\section{Case Study and Implementation Architecture}

For the purpose of implementing such context-aware delivery service of contextual metrics, we propose (Figure 14), a service oriented architecture (SOA) for GeoBI location-based and context-based reasoning and analysis. Service oriented architectures ease abstraction, reusability and interoperability, and are more suitable for mobile computing than client/server architectures [61]. The proposed architecture operates as follows:

The context-aware service is responsible for handling "push" and "pull" requests. It regularly senses and acquires contextual information from the user's mobile context through various sensors (e.g. GPS, Gyroscop, log-

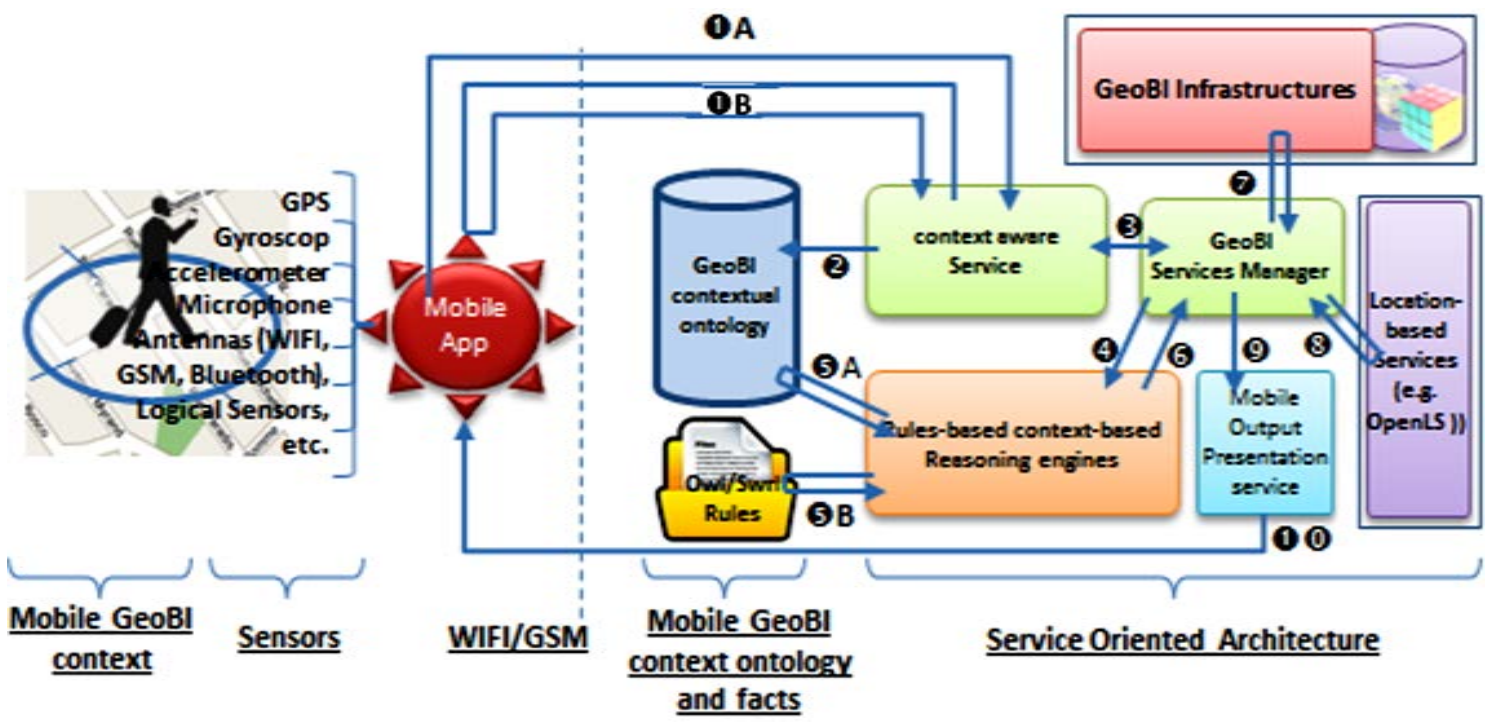

Figure 14. Service oriented architecture (SOA) for GeoBI location-based and context-based reasoning and analysis. 
ical sensors, etc.) available on the mobile device on which a context-aware mobile GeoBI application is running.

"Pull" requests are issued by the mobile user, while "push" requests are auto-generated by the context-aware service based on preconfigured indications (e.g. when a salesman enters a new location-e.g. district-, send him the list of top 5 best-selling products in that location). As a case study, let consider that salesman Steve wants to get the sales performed by the supervisors of the best performing salesman (e.g. John) of this quarter in the sales zone he is located in (see Section 2.1).

1) Once Steve submits his request, the mobile application captures his current context (e.g. location, agenda, etc.) and sends the request and the captured contextual information to the context-aware service $(1 \mathrm{~A})$. The context-aware service handles these and may call back the mobile application (1B) to get additional contextual information if necessary (e.g. if the submitted request for enriched HD BI graphics that require the mobile device battery level to be greater than $10 \%$, the battery current level has to be retrieved).

2) The contextual information database is updated with acquired information to keep contextual information up-to-date. The GeoBI context ontology database is supposed to be fed with facts.

3) The context-aware service calls the GeoBI Services Manager (GeoBI-SM) by indicating the GeoBI service requested by the user, and by transmitting the request and captured contextual information. GeoBI-SM launches the requested service (e.g. GeoBI sales delivery service) which builds an ontological request-e.g. by using SPARQL [62] - to retrieve or derive from the GeoBI context ontology, all contextual data (e.g. current sales zone, all supervisors of John) required to select the requested sales. A BPEL (Business Process Execution Language) process can be used to orchestrate and indicate how and when the services should be called [63] [64].

4) GeoBI-SM submits the request to the context-based reasoning engines.

5A) and 5B) Reasoning engines-e.g. Jena [65] — load the GeoBI context ontology and facts into their knowledge bases, and then infer to get the requested data. The reasoning process may be enriched with semantic rules-e.g. SWRL [66] rules-that can be written by semantic data specialists to into take account business rules (e.g. the relation of owning a company is transitive). These rules might also indicate the minimum threshold of certainty level defined by the company (e.g. 0.6) from which a given contextual information can be accepted as valid.

6) The reasoning results (e.g. Steve's current sales zone from his current position, all supervisors of John) are sent back to GeoBI-SM.

7) GeoBI-SM operates a context-based request of GeoBI data (e.g. sales) from GeoBI infrastructures by using contextual information returned by reasoning engines as parameters (e.g. select sales performed by the list of supervisors within the current sales zone).

8) Location-based services and mapping services may be called by the GeoBI-SM to display maps and locations (e.g. display in background, places where sales were performed).

9) GeoBI-SM communicates output data to the Mobile Output Presentation service which renders and sends it to the mobile GeoBI application in response to the user's push/pull request.

A prototype is currently under development and will be published in future papers.

\section{Conclusions and Future Work}

This paper has supplied an extended and detailed OWL-based mobile GeoBI context ontology to provide context-aware applications and users with relevant contextual information and context-based reasoning capabilities. Throughout the article, we have successively: 1) highlighted the inferential power of OWL ontologies and the need for GeoBI context-based reasoning and OWL modeling; 2) presented and integrated spatial and temporal pictograms and properties into Cmap Tools and OWL to suitably design spatio-temporal concepts; 3) proposed a multilevel top-level mobile GeoBI context ontology which provides a generic overview of context stakeholders and highlights its hierarchical multilevel structure which matches with human cognition and contextual information aggregation; 4) provided for each context dimension (personal, business, technological, spatial, social, and environmental contexts) a detailed context ontology; 5) tackled context acquisition and quality issues; and finally, 6) proposed an implementation architecture.

Based on this work, further work is dealing with the development of a functioning prototype. Future works will introduce the notion of contextual metrics (e.g. revenue per capita) besides contextual information, and deal with integrating them into the OWL-based mobile GeoBI context ontology proposed in this paper in order to boost its capacity to support context-reasoning and contextual business analysis. 


\section{References}

[1] Franklin, C. (1992) An Introduction to Geographic Information Systems: Linking Maps to Databases. Database, 15, 13-21.

[2] Urry, J. (2002) Mobility and Proximity. Sociology, 36, 255-274. http://dx.doi.org/10.1177/0038038502036002002

[3] Bao, T., Cao, H., Chen, E., Tian, J. and Xiong, H. (2012) An Unsupervised Approach to Modeling Personalized Contexts of Mobile Users. Knowledge and Information Systems, 31, 345-370. http://dx.doi.org/10.1007/s10115-011-0417-1

[4] Chen, H., Finin, T. and Joshi, A. (2003) An Intelligent Broker for Context-Aware Systems. Adjunct Proceedings of Ubicomp, Vol. 3, Seattle, 12-15 October 2003, 183-184.

[5] Diallo, B.A.A., Badard, T., Hubert, F. and Daniel, S. (2012) Mobile and Context-Aware GeoBI Applications: A Multilevel Model for Structuring and Sharing of Contextual Information. Journal of Geographic Information System (JGIS), 4, 425-443. http://dx.doi.org/10.4236/jgis.2012.45048

[6] Wang, X.H., Zhang, D.Q., Gu, T. and Pung, H.K. (2004) Ontology Based Context Modeling and Reasoning Using OWL. 2nd IEEE Conference on Pervasive Computing and Communications (PerCom 2004) Workshop on Context Modeling and Reasoning, Orlando, 14-17.

[7] Gu, T., Wang, X., Pung, H. and Zhang, D. (2004) An Ontology-Based Context Model in Intelligent Environments. Communication Networks and Distributed Systems Modeling and Simulation Conference, San Diego.

[8] Antoniou, G. and van-Harmelen, F. (2004) Web Ontology Language: OWL. Handbook on Ontologies, Springer, Berlin, New York, 67-92.

[9] Hayes, P., Eskridge, T.C., Mehrotra, M., Bobrovniko, D., Reichherzer, T. and Saavedra, R. (2005) COE: Tools for Collaborative Ontology Development and Reuse. Knowledge Capture Conference (K-CAP) 2005, Banff, 2-5 October 2005, 1-6.

[10] Parreiras, S.F. and Staab, S. (2010) Using Ontologies with UML Class-Based Modeling: The Two Use Approach. Data \& Knowledge Engineering, 69, 1194-1207. http://dx.doi.org/10.1016/j.datak.2010.07.009

[11] Straeten, R.V.D., Mens, T., Simmonds, J. and Jonckers, V. (2003) Using Description Logic to Maintain Consistency between UML Models. UML 2003-The Unified Modeling Language, Modeling Languages and Applications, 6th International Conference, San Francisco.

[12] Bodeveix, J., Millan, T., Percebois, C., Camus, C., Bazex, P. and Feraud, L. (2002) Extending OCL for Verifying UML Models Consistency. UML 2002: Workshop on Consistency Problems in UML-Based Software Development.

[13] Bédard, Y. (1999) Principles of Spatial Database Analysis and Design. GIS: Principles, Techniques, Applications \& Management, 2nd Edition. Wiley, New York, 413-424.

[14] Yong, L. and Chen, S. (2011) Visual Modeling and Representations of Spatiotemporal Transportation Data: An Object-Oriented Approach. 2011 International Symposium on Computer Science and Society (ISCCS), Kota Kinabalu, 16-17 July 2011, 218-222.

[15] Brodeur, J. (2012) Geosemantic Interoperability and the Geospatial Semantic Web. Handbook of Geographic Information, Springer Heidelberg, Berlin, 291-310.

[16] W3C (2007) W3C Geospatial Vocabulary. http://www.w3.org/2005/Incubator/geo/XGR-geo-20071023/\#owl

[17] Tversky, B. (2003) Structures of Mental Spaces: How People Think about Space. Environment and Behaviour, 35, 6680.

[18] Graves, M., Constabaris, A. and Brickley, D. (2007) FOAF: Connecting People on the Semantic Web. Cataloging and Classification Quarterly, 43, 191-202. http://dx.doi.org/10.1300/J104v43n03_10

[19] Costa, P. and McCrae Jr., R. (1992) Revised NEO Personality Inventory (NEO-PI-R) and NEO Five-Factor Inventory (NEO-FFI) Manual. Psychological Assessment Resources, Lutz.

[20] Salgado, J. (2003) Predicting Job Performance Using FFM and Non-FFM Personality Measures. Journal of Occupational and Organizational Psychology, 76, 323-346. http://dx.doi.org/10.1348/096317903769647201

[21] Yuanchao, M., Bin, X., Yin, B., Guodong, S. and Zhu, R. (2012) Daily Mood Assessment Based on Mobile Phone Sensing. Ninth International Conference on Wearable and Implantable Body Sensor Networks (BSN), London, 9-12 May 2012, 142-147.

[22] Chittaranjan, G., Blom, J. and Gatica-Perez, D. (2011) Who’s Who with Big-Five: Analyzing and Classifying Personality Traits with Smartphones. International Symposium on Wearable Computers (ISWC), Bonn, 23-27 October 2011, 29-36.

[23] Luhn, H. (1958) A Business Intelligence System. IBM Journal of Research and Development, 2, 314-319.

[24] Novakovic, D. and Huemer, C. (2014) A Survey on Business Context. Intelligent Computing, Networking, and Informatics, Springer India, 199-211. 
[25] Douma, S. and Schreuder, H. (2013) Economic Approaches to Organizations. 5th Edition, Pearson Education Limited, Harlow.

[26] Dale, B.G., Van Der Wiele, T. and Van Iwaarden, J. (2013) Managing Quality. John Wiley \& Sons, Hoboken.

[27] Meyer, K.E., Mudambi, R. and Narula, R. (2011) Multinational Enterprises and Local Contexts: The Opportunities and Challenges of Multiple Embeddedness. Journal of Management Studies, 48, 235-252.

[28] Grant, R.M. (2010) Contemporary Strategy Analysis and Cases: Text and Cases. John Wiley \& Sons, Hoboken.

[29] Andrews, K.R. (1970) The Concept of Corporate Strategy. Dow Jones-Irwin, Homewood.

[30] Kindberg, T., Bevan, C., O’Neill, E., Mitchell, J., Grimmett, J. and Woodgate, D. (2009) Authenticating Ubiquitous Services: A Study of Wireless Hotspot Access. 11th International Conference on Ubiquitous Computing (Ubicomp’09), New York.

[31] Goodchild, M.F., Yuan, M. and Cova, T.J. (2007) Towards a General Theory of Geographic Representation in GIS. International Journal of Geographical Information Science, 21, 239-260. http://dx.doi.org/10.1080/13658810600965271

[32] Murray, A.T. (2010) Advances in Location Modeling: GIS Linkages and Contributions. Journal of Geographical Systems, 12, 335-354. http://dx.doi.org/10.1007/s10109-009-0105-9

[33] Rosenberg, M. (2014) The Five Themes of Geography. http://geography.about.com/od/teachgeography/a/5themes.htm

[34] Jones, C. (2008) Modelling Vague Places with Knowledge from the Web. International Journal of Geographical Information Science, 22, 1045-1065.

[35] Kelm, P., Murdock, V., Schmiedeke, S., Schockaert, S., Serdyukov, P. and Van Laere, O. (2013) Georeferencing in Social Networks. Social Media Retrieval, 115, 115-142. Springer-Verlag, London. http://dx.doi.org/10.1007/978-1-4471-4555-4_6

[36] Callan, R.J. and Kyndt, J. (2001) Business Travellers’ Perception of Service Quality: A Prefatory Study of Two European City Centre Hotels. International Journal of Tourism Research, 3, 313-323. http://dx.doi.org/10.1002/jtr.333

[37] Fawzy, A. (2010) Business Travelers' Accommodation Selection: A Comparative Study of Two International Hotels in Cairo. International Journal of Hospitality and Tourism Administration, 11, 138-156. http://dx.doi.org/10.1080/15256481003732790

[38] Tarlow, P. (2009) Tourism Safety and Security. The SAGE Handbook of Tourism Studies, SAGE Publications Ltd., London, 464-480.

[39] Perry, M., O’Hara, K., Sellen, A., Brown, B. and Harper, R. (2001) Dealing with Mobility: Understanding Access Anytime, Anywhere. ACM Transactions on Computer-Human Interaction, 8, 323-347. http://dx.doi.org/10.1145/504704.504707

[40] Raento, M., Oulasvirta, A., Petit, R. and Toivonen, H. (2005) ContextPhone: A Prototyping Platform for Context-Aware Mobile Applications. IEEE Pervasive Computing, 4, 51-59. http://dx.doi.org/10.1109/MPRV.2005.29

[41] Nicolai, T., Yoneki, E., Behrens, N. and Kenn, H. (2006) Exploring Social Context with the Wireless Rope. Lecture Notes in Computer Science, 4277, 874-883.

[42] Zack, M.H. and McKenney, J.L. (1995) Social Context and Interaction in Ongoing Computer-Supported Management Groups. Organization Science, 6, 394-422. http://dx.doi.org/10.1287/orsc.6.4.394

[43] Ferris, G., Arthur, M., Berkson, H., Kaplan, D., Harrel-Cook, G. and Frink, D. (1998) Toward a Social Context Theory of the Human Resource Management Organization Effectiveness Relationship. Human Resource Management Review, 8, 235-264. http://dx.doi.org/10.1016/S1053-4822(98)90004-3

[44] Carroll, A. and Bucholtz, A. (2008) Ethics and Stakeholder Management. Business and Society with Infotrac, Mason.

[45] Boiral, O. (2006) Global Warming: Should Companies Adopt a Proactive Strategy? Long Range Planning, 39, 315-330. http://dx.doi.org/10.1016/j.lrp.2006.07.002

[46] Wang, H., Lymberopoulos, D. and Liu, J. (2014) Local Business Ambience Characterization through Mobile Audio Sensing. Proceedings of the 23rd International Conference on World Wide Web, Seoul, 7-11 April 2014, 293-304.

[47] Barry, R.G. and Chorley, R.J. (2009) Atmosphere, Weather and Climate. Routledge, London.

[48] McBride, A.C., Dale, V.H., Baskaran, L.M., Downing, M.E., Eaton, L.M., Efroymson, R.A. and Storey, J.M. (2011) Indicators to Support Environmental Sustainability of Bioenergy Systems. Ecological Indicators, 11, 1277-1289. http://dx.doi.org/10.1016/j.ecolind.2011.01.010

[49] Mace, G.M., Norris, K. and Fitter, A.H. (2012) Biodiversity and Ecosystem Services: A Multilayered Relationship. Trends in Ecology \& Evolution, 27, 19-26.

[50] Beder, S. (2013) Environmental Principles and Policies: An Interdisciplinary Introduction. Routledge, London.

[51] Henricksen, K. and Indulska, J. (2004) Modelling and Using Imperfect Context Information. 1st Workshop on Context 
Modeling and Reasoning, PerCom'04, Orlando, 14-17 March 2004, 33-37.

[52] Ranganathan, A., Al-Muhtadi, J. and Campbell, R. (2004) Reasoning about Uncertain Contexts in Pervasive Computing Environments. IEEE Pervasive Computing, 3, 62-70.

[53] Bettini, C., Brdiczka, O., Henricksen, K., Indulska, J., Nicklas, D., Ranganathan, A. and Riboni, D. (2010) A Survey of Context Modelling and Reasoning Techniques. Pervasive and Mobile Computing, 6, 161-180. http://dx.doi.org/10.1016/j.pmcj.2009.06.002

[54] Manzoor, A., Truong, H.L. and Dustdar, S. (2014) Quality of Context: Models and Applications for Context-Aware Systems in Pervasive Environments. The Knowledge Engineering Review, 29, 154-170. http://dx.doi.org/10.1017/S0269888914000034

[55] McKeever, S., Ye, J., Coyle, L. and Dobson, S. (2009) A Context Quality Model to Support Transparent Reasoning with Uncertain Context. Lecture Notes in Computer Science, 5786, 65-75. http://dx.doi.org/10.1007/978-3-642-04559-2_6

[56] Helaoui, R., Riboni, D., Niepert, M., Bettini, C. and Stuckenschmidt, H. (2012) Towards Activity Recognition Using Probabilistic Description Logics. Workshops at the 26th AAAI Conference on Artificial Intelligence on Activity Context Representation: Techniques and Languages, Toronto, 22-26 July 2012, 26-31.

[57] Frez, J., Baloian, N., Zurita, G. and Pino, J.A. (2014) Dealing with Incomplete and Uncertain Context Data in Geographic Information Systems. IEEE 18th International Conference on Computer Supported Cooperative Work in Design (CSCWD), Hsinchu, 21-23 May 2014, 129-134.

[58] Chahuara, P., Portet, F. and Vacher, M. (2012) Context Aware Decision System in a Smart Home: Knowledge Representation and Decision Making Using Uncertain Contextual Information. The 4th International Workshop on Acquisition, Representation and Reasoning with Contextualized Knowledge (ARCOE-12), Montpellier, 28 August 2012, 5264.

[59] Sottara, D., Mello, P. and Proctor, M. (2010) A Configurable Rete-OO Engine for Reasoning with Different Types of Imperfect Information. IEEE Transactions on Knowledge and Data Engineering, 22, 1535-1548. http://dx.doi.org/10.1109/TKDE.2010.125

[60] McKeever, S. (2011) Recognising Situations Using Extended Dempster-Shafer Theory. Doctoral Dissertation, National University of Ireland, Dublin.

[61] Badard, T., Bédard, Y., Hubert, F., Bernier, E. and Dubé, É. (2008) Web Services Oriented Architectures for Mobile SOLAP Applications. International Journal of Web Engineering and Technology (IJWET), 4, 434-464. http://dx.doi.org/10.1504/IJWET.2008.019943

[62] Quilitz, B. and Leser, U. (2008) Querying Distributed RDF Data Sources with SPARQL. The Semantic Web: Research and Applications, Springer, Heidelberg, 524-538.

[63] Akram, A., Meredith, D. and Allan, R. (2006) Evaluation of BPEL to Scientific Workflows. 6th IEEE International Symposium on Cluster Computing and the Grid, CCGRID 06, 1, 269-274.

[64] Pautasso, C. (2009) RESTful Web Service Composition with BPEL for REST. Data \& Knowledge Engineering, 68, 851-866.

[65] Carroll, J.J., Dickinson, I., Dollin, C., Reynolds, D., Seaborne, A. and Wilkinson, K. (2004) Jena: Implementing the Semantic Web Recommendations. WWW 2004 13th Conference on World Wide Web, New York, 19-21 May 2004, 74-83.

[66] O’Connor, M., Knublauch, H., Tu, S. and Musen, M. (2005) Writing Rules for the Semantic Web Using SWRL and Jess. Lecture Notes in Computer Science, 3729, 974-986. 
Scientific Research Publishing (SCIRP) is one of the largest Open Access journal publishers. It is currently publishing more than 200 open access, online, peer-reviewed journals covering a wide range of academic disciplines. SCIRP serves the worldwide academic communities and contributes to the progress and application of science with its publication.

Other selected journals from SCIRP are listed as below. Submit your manuscript to us via either submit@scirp.org or Online Submission Portal.
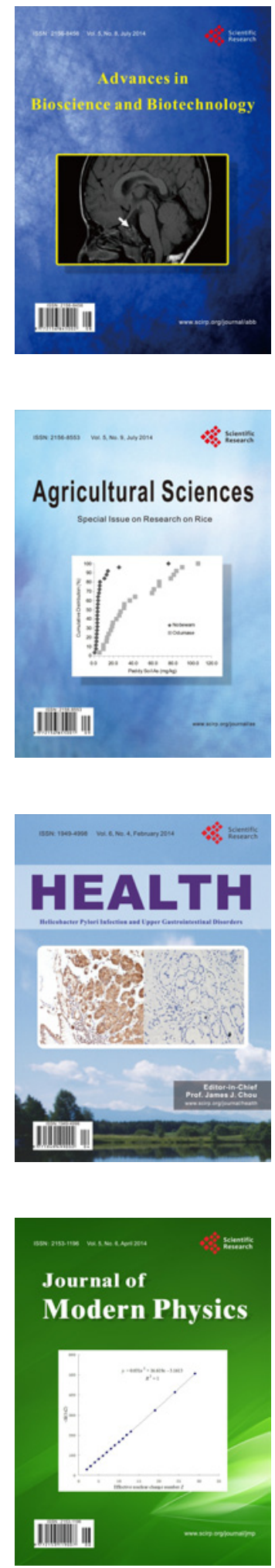
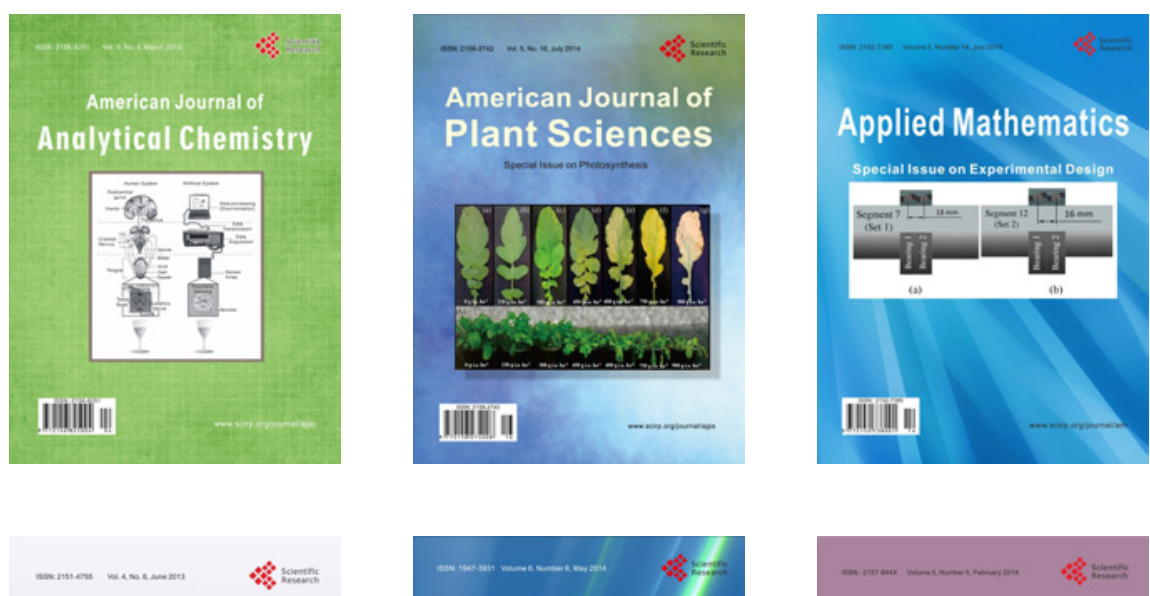

Creative Education
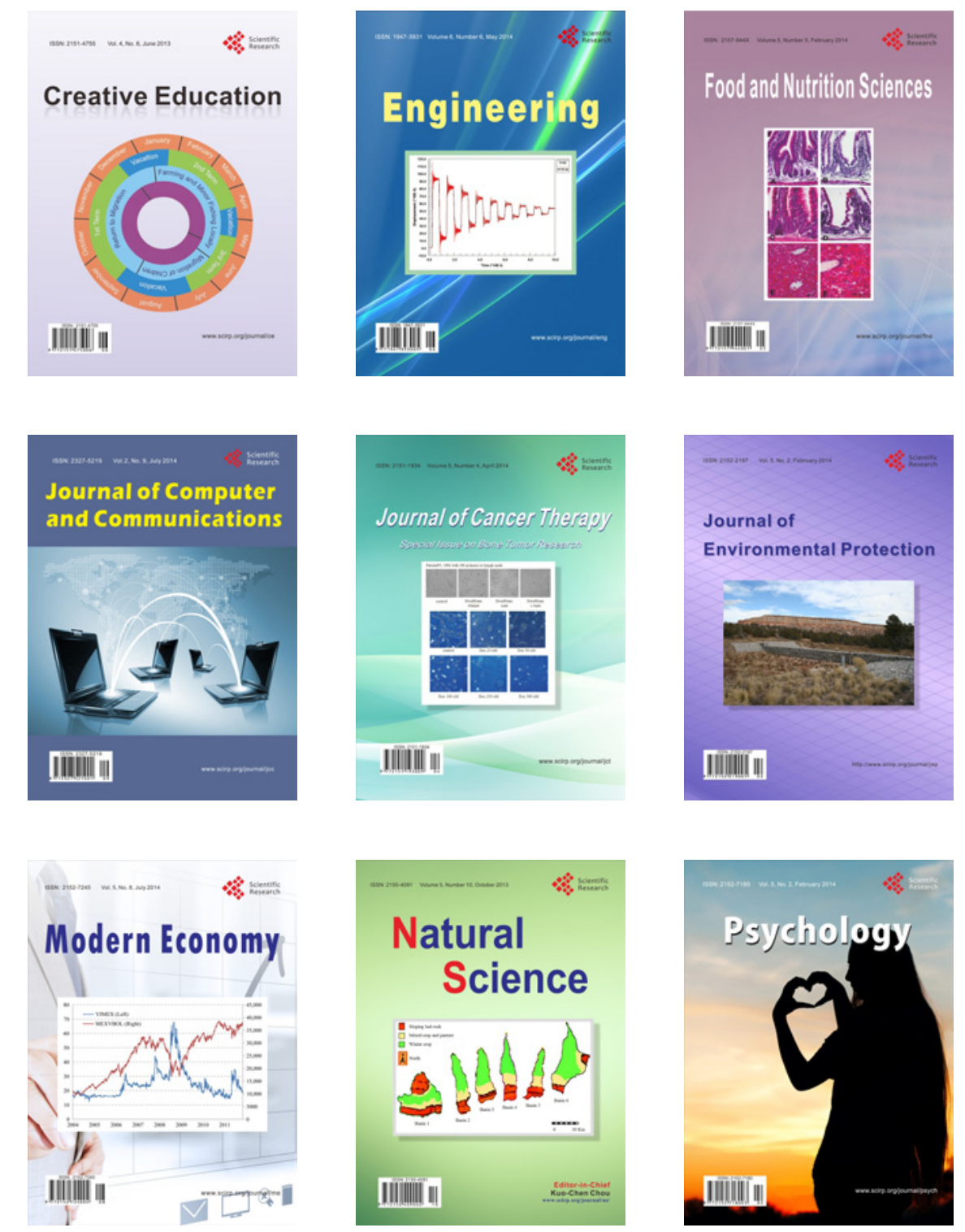